\title{
Diversity of mantids (Dictyoptera: Mantodea) of Sangha-Mbaere Region, Central African Republic, with some ecological data and DNA barcoding
}

\author{
Nicolas Moulin', Thibaud Decaëns², Philippe Annoyer ${ }^{3}$
}

1 82, route de l'école, Hameau de Saveaumare, 76680 Montérolier, France.

2 Centre d'Ecologie Fonctionnelle et Evolutive, UMR 5175, CNRS, Université de Montpellier, 1919 Route de Mende, 34293 Montpellier Cedex 5, France. 3 Insectes du Monde Sabine, 09230 Sainte Croix de Volvestre, France.

Corresponding author: Nicolas Moulin (nmentomo@gmail.com)

Academic editor: Matan Shelomi | Received 27 July 2017 | Accepted 21 September 2017 | Published 24 November 2017

http://zoobank.org/DBD570D6-4A5F-4D5F-8C59-4A228B2217FF

Citation: Moulin N, Decaëns T, Annoyer P (2017) Diversity of mantids (Dictyoptera: Mantodea) of Sangha-Mbaere Region, Central African Republic, with some ecological data and DNA barcoding. Journal of Orthoptera Research 26(2): 117-141. https://doi.org/10.3897/jor.26.19863

\begin{abstract}
This study aims at assessing mantid diversity and community structure in a part of the territory of the Sangha Tri-National UNESCO World Heritage Site in the Central African Republic (CAR), including the special forest reserve of Dzanga-Sangha, the Dzanga-Ndoki National Park. The study area is located in the biome of the dense tropical rainforest of the Congo Basin, the second largest area of tropical forest behind the Amazon basin, and one of the last regions on earth where pristine forest ecosystems persist. Between 1984 and 2012, eight collecting campaigns were conducted by P. Annoyer, M. Loubes and S. Danflous, with the aim of documenting invertebrate diversity in this remote and poorly studied area. Mantids were collected in different habitats and microhabitats using four harvesting techniques: visual hunting (threshing, mowing), active search in the trees, autonomous UV collecting (Remote Canopy Trap) and classical light trapping with mercury light. Community patterns were assessed using rarefaction curves and diversity estimators (ACE). Specimens $(n=25)$ were also found at the National Museum of Natural History (MNHN) of Paris. Seventy-one species of Mantodea are recorded from Sangha-Mbaere Region in the CAR from 1232 specimens. This is the first synthesis published about Mantodea in CAR. Some new species are present in the genera Cataspilota, Galepsus and Chlidonoptera. Taxonomic revisions of these genera are in progress. Some genera need global revision like Plistospilota, Miomantis, Entella, and Galepsus. This type of work is the first on the mantids in Central Africa since the various inventories carried out in the sixties and seventies, which were mainly based on lists of species. The contribution of data on ecology and biogeography is a novelty.
\end{abstract}

\section{Key words}

Congo Basin, DNA barcoding, ecology, Praying Mantis, sampling methods, species richness, tropical rainforest

\section{Introduction}

Currently, over 2,400 valid species of Mantodea have been recorded worldwide (Giglio-Tos 1927, Ehrmann 2002, Otte and Spearman 2005, Otte et al. 2017). Since 2005, several generic revisions have been published (Roy 2009, 2010, Roy 2013a, 2013b,
Roy and Stiewe 2014, Tedrow et al. 2014, Svenson et al. 2015). In Africa, only surveys by R. Roy, in the years 1960 to 1980, provided distribution records of Mantodea from several African countries (Ivory Coast, Gabon, Ghana, Guinea, Senegal, etc.); and those of A. Kaltenbach in 1996 and 1998 provided records from South Africa. During all these years, many research stations were in operation, and many samples were collected there (La Maboké-Boukoko in CAR, for example). They are, for the most part, unpublished. More recently, a team published results from a survey in Rwanda (Tedrow et al. 2015). According to this research, the number of African Mantodea is estimated to be over 1000 valid species.

The rainforests of Central Africa are the largest rainforest block after the Amazon basin (UNEP 2008). This is one of the last undisturbed biological environments. Evergreen forests of Central Africa, with an area of about 1.62 million square kilometers (36\% of the country surface), represent an exceptional natural heritage (Basset 2001, Basset et al. 2003, Lowman and Rinker 2004, Vande Weghe 2004, Dial et al. 2006, UNEP 2008). They host a significant proportion of the world's biodiversity, and play an important role in climate regulation and carbon sequestration (Ngatoua 1997, Aveling and Debonnet 2010).

Sangha-Mbaere Region is located in the Southwest of the Central African Republic (CAR) (Fig. 1). It is named in reference to the Sangha and Mbaere Rivers, which both represent the main elements of the regional hydrographic network. This region covers a total area of $19412 \mathrm{~km}^{2}$ and is extensively covered by tropical rainforests in the southern triangular-shaped part of the region. It contains several natural lakes of different size (Massa 2013, de Wasseige et al. 2014). Two main types of forests have been distinguished: a) dryland forests present open, mixed canopy dominated by Sterculiaceae and Ulmaceae, generally with a dense understory vegetation of Maranthaceae and Zingiberaceae (Aveling and Debonnet 2010, Massa 2013), b) semi-evergreen forests contain swamp-forest areas along the rivers, and closed-canopy, monodominant Gilbertiodendron dewevrei formations, with trees over 50 meters high (UNEP 2008). The northern part of the Region, north of Nola, is currently undergoing a dramatic process of deforesta- 


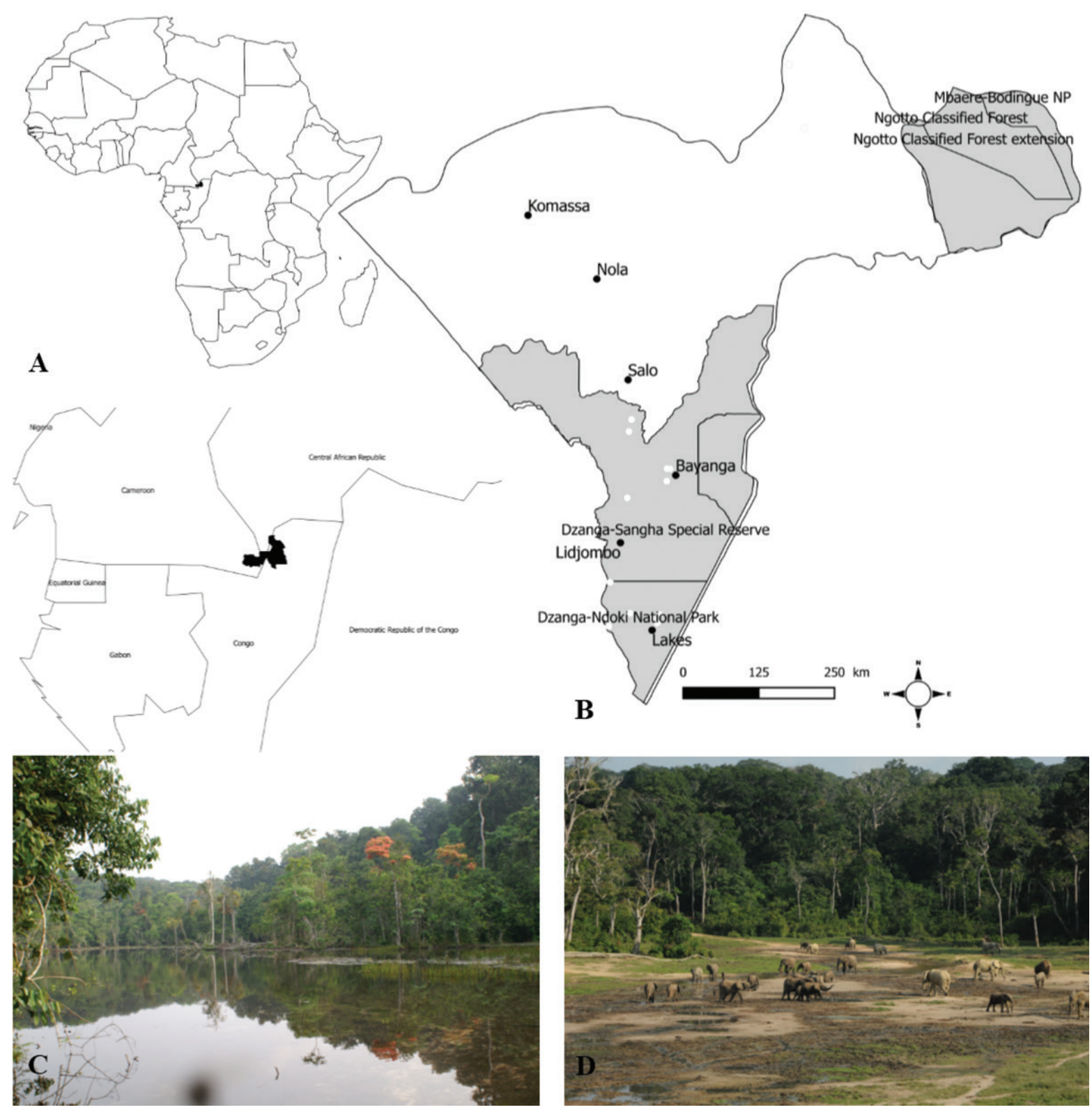

Fig. 1. Sangha-Mbaere Region in Central African Republic. A. Location of Tri National Sangha area in Central Africa; B. Location of principal sampling stations and largest towns. Black dots: Main city in Sangha-Mbaere Region. White dots: Main collection areas. Sources: http://www.diva-gis.org adminstrative area, Sangha-Mbaere Region; C. Illustration of the habitats studied; D. Primary forest of CAR, Dzanga Bai with forest elephants.

tion. In the extreme southwest, Dzanga-Ndoki National Park is part of the Sangha Tri-National (TNS), a UNESCO World Heritage Site which covers an area of 4420000 ha and includes three national parks: Loboké (Cameroon), Nouabalé-Ndoki (Congo) and Dzanga-Ndoki (CAR) (Ngatoua 1997, Massa 2013). Another National Park, the Mbaéré-Bodingué National Park, was recently created near Mbaere River, including the beautiful Ngotto Forest (Brugiere et al. 2005, Ndarata Massanguet 2012). This part of the region is a very rich sanctuary of biodiversity, which is the focus of recent scientific investigations.

Here, we report on the diversity of the Mantodea (Insecta: Dictyoptera) found within Sangha-Mbaere Region since 1984. Couturier et al. (1986) described that some insect collections remained in the research center of Boukoko and La Maboké, CAR, after de- parture of expatriate researchers. Unfortunately, poor conservation of these specimens did not permit us to study them. Currently, the vast majority of Mantodea from CAR are conserved at the Muséum national d'Histoire naturelle of Paris. Nevertheless, mantids in this collection are mainly native to the Lobaye, CAR, region. These specimens are disregarded in the current work, but will be the focus for a future publication.

Our review covers all species found on decades of collecting trips through Sangha-Mbaere Region. We discuss the ecological traits and taxonomic status of the Mantodea of this under-sampled region. Some specimens are not identified to species. They will be described in other papers, which will include generic revision (e.g. Cataspilota, Chlidonoptera, Plistospilota). Some others need more taxonomic research (e.g. Miomantis, Entella, Galepsus). 

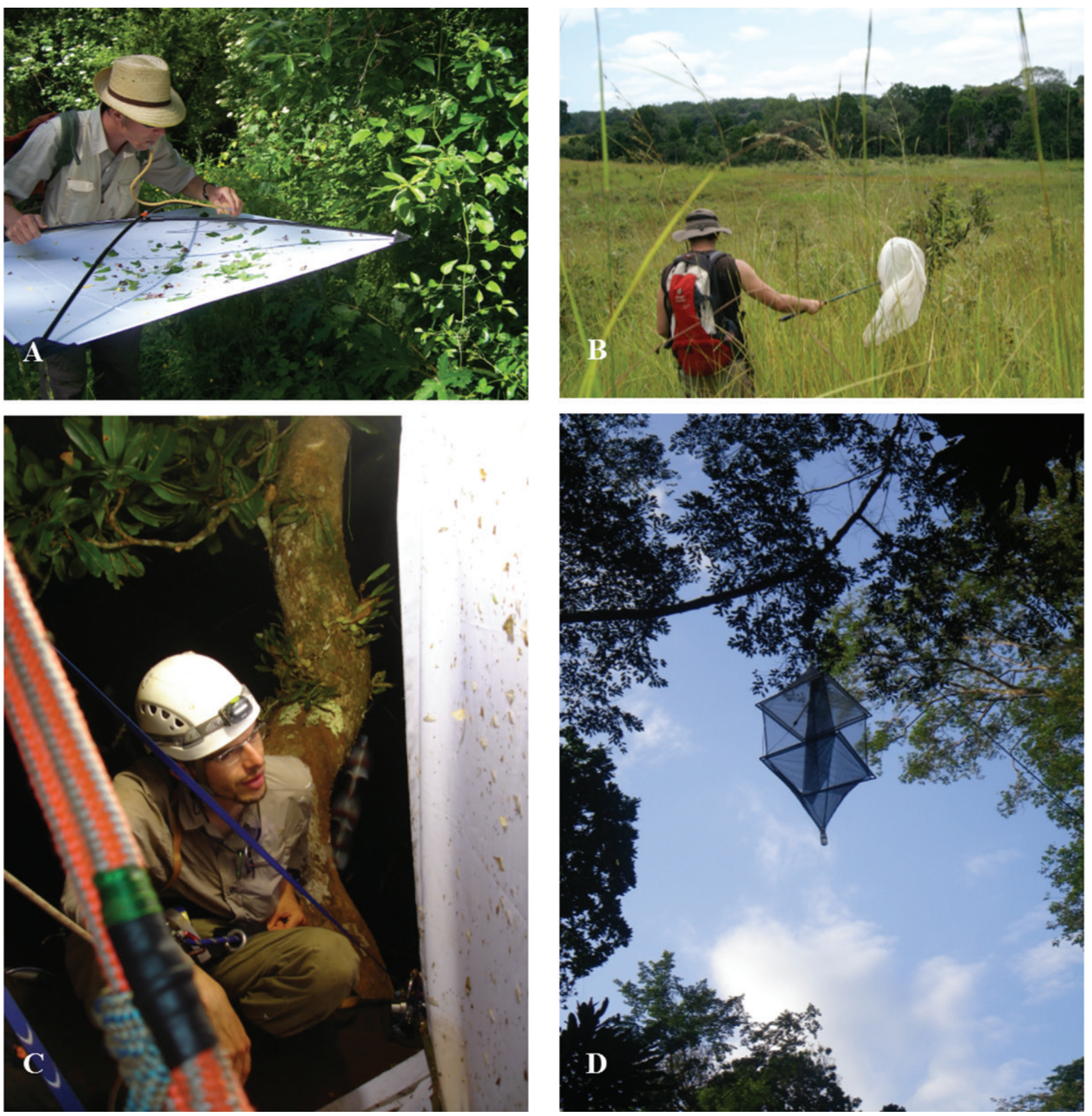

Fig. 2. Illustration of some sampling methods used during surveys in CAR. A. Beating sheet; B. Sweep net; C. Tree climbing; D. Remote Canopy Trap.

\section{Material and methods}

Specimen sampling.-Since 1984, several trips were made to the Sangha-Mbaere Region to collect insects (with a focus on Lepidoptera: Rhopalocera). In 2008, the expedition, named Epiphyte 2008, visited the Dzanga-Sangha Reserve. In November 2010, the one-month long expedition was a reconnaissance mission which paved the way for a larger expedition of two months, called SANGHA "Biodiversité en Terre Pygmée", in January-March 2012. The last two expeditions (2010 and 2012) were organized in the Dzanga-Ndoki National Park.

Most specimens were collected using light traps consisting of a white sheet illuminated by a $250 \mathrm{~W}$ mercury vapor bulb. In 2010 and 2012, light traps (Mercury Vapor lamps, economics lamps, neons) were complemented by other sampling procedures, including visual collecting during tree climbing, night and day research in understory vegetation, remote canopy trap, and aerial interception trap (Fig. 2). Historically, scientific research on the praying mantids made use of their attraction to light (Mercury Vapor). Lamps producing ultraviolet light are suspended in front of vertical white sheets. However, mostly males are attracted and very few females, and only from the very few species that are attracted to light. To complete the sampling, mowing, beating vegetation and visual searching for individuals during the day were also performed. Using these techniques, many nymph and female individuals were collected.

Specimens were killed using cyanide vapour jars, dried by wood fire smoke and preserved in paper bags or on cotton wrap. All specimens were then studied in the laboratory of Nicolas Moulin.

Taxonomic assignations. - Identifications were done following the updated taxonomy in the Mantodea Species File website (Otte et 

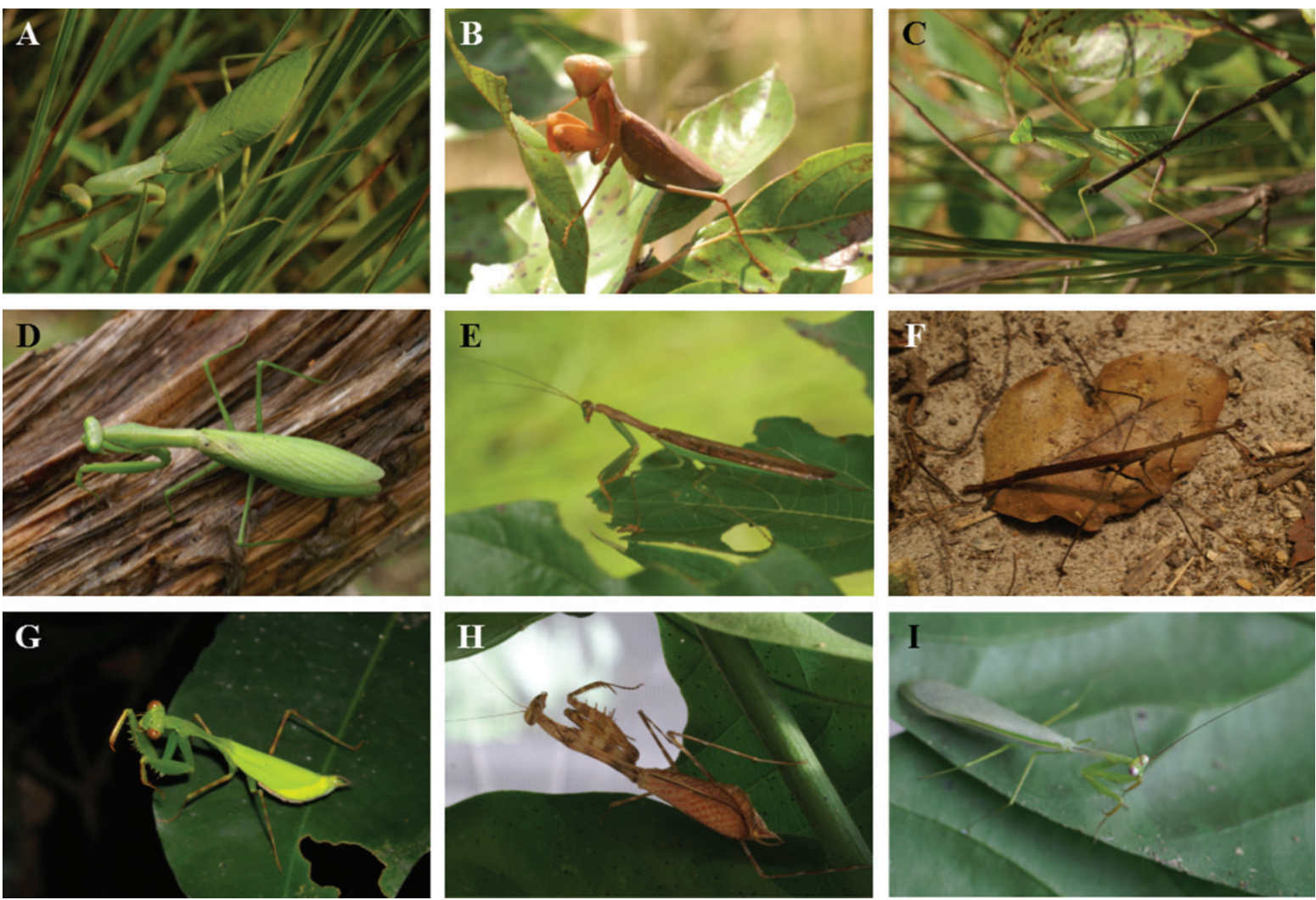

Fig. 3. Live habitus. A. Sphodromantis lineola pinguis (green female) from LNP; B. Sphodromantis lineola pinguis (brown female) from LNP; C. Sphodromantis lineola pinguis (green male) from LNP; D. Alalomantis muta (female) from CMNP; E. Polyspilota aeruginosa (male) from LNP; F. Macrodanuria elongata (male) from DNNP; G. Miomantis preussi (green female) from DNNP; H. Miomantis preussi (brown female) from DNNP; I. Miomantis preussi (male) from DNNP.

al. 2017) and in recent works on praying mantis molecular phylogenetics (Svenson and Whiting 2009, Wieland 2013, Roy 2014, Svenson et al. 2015). The identifications were made on the basis of habitus morphology (Figs 3-4), color patterns, foreleg framing and genitalia morphology.

Ecological analysis. - An occurrence table consisting of 71 species and 1232 individuals was constructed, where the locality, habitat and sampling method were documented. This table was then used to compute observed richness (calculated as the total number of species observed in a given locality or habitat, or for a given sampling method) and an estimation of the theoretical species richness using the ACE diversity estimator using the 'Vegan' Package for R v. 2.7.2. (Oksanen et al. 2008). Additionally, the completeness of the sampling at a regional scale was assessed by computing a rarefaction / extrapolation curve using the iNEXT package (Chao and Jost 2012). On the ground, distinctions were made between sampling in herbaceous vegetation $(<1 \mathrm{~m})$, shrubs (between $1 \mathrm{~m}$ and $3 \mathrm{~m}$ ) and trees (between $3 \mathrm{~m}$ and canopy).

DNA barcoding. - DNA barcoding, the analysis of a standardized segment of the mitochondrial cytochrome c oxidase subunit I (COI) gene, was performed on a representative selection of specimens (171, selected by diversity criteria). Tissues were sent to the Canadian Center for DNA Barcoding (CCDB) at the University of Guelph for DNA extraction, polymerase chain reaction (PCR) and sequencing. PCR was performed using the PCR primers $\mathrm{C}_{-}$ LepFolF/C_LepFolR. Data are currently managed under projects: "Mantodea of Gabon - Project 1 [ECOTROP 2014]", "Mantodea of Gabon - Project 2 [ECOTROP 2011]", "DNA Barcoding Mantodea - Collection N. Moulin" at Barcode of Life Data Systems (BOLD, Biodiversity Institute of Ontario, Canada; boldsystems. org). Kimura-2-parameter (K2P) distances were calculated using the BOLD 4.0 interface (Ratnasingham and Hebert 2007). Sequences were then analyzed and trees constructed using BOLD 4.0 interface.

Deposition of specimens. - All specimens are, at the moment, in the "Insectes du Monde" collection (Ph. Annoyer, Sabine, France) and in the Research Collection of Nicolas Moulin (Montérolier, France).

Abbreviations used in this paper:

BOLD Barcode of Life Project, Biodiversity Institute of Ontario; IDM Insectes du Monde Collection, P. Annoyer, Sainte-Croixde-Volvestre;

RCNM Research Collection of Nicolas Moulin, Montérolier;

MSC Martin Stiewe Collection, Berkshire;

DNNP Dzanga-Ndoki National Park, Central African Republic;

LNP Lope National Park, Gabon;

CMNP Cristal Mountains National Park, Gabon;

NM Nicolas Moulin;

PA Philippe Annoyer. 

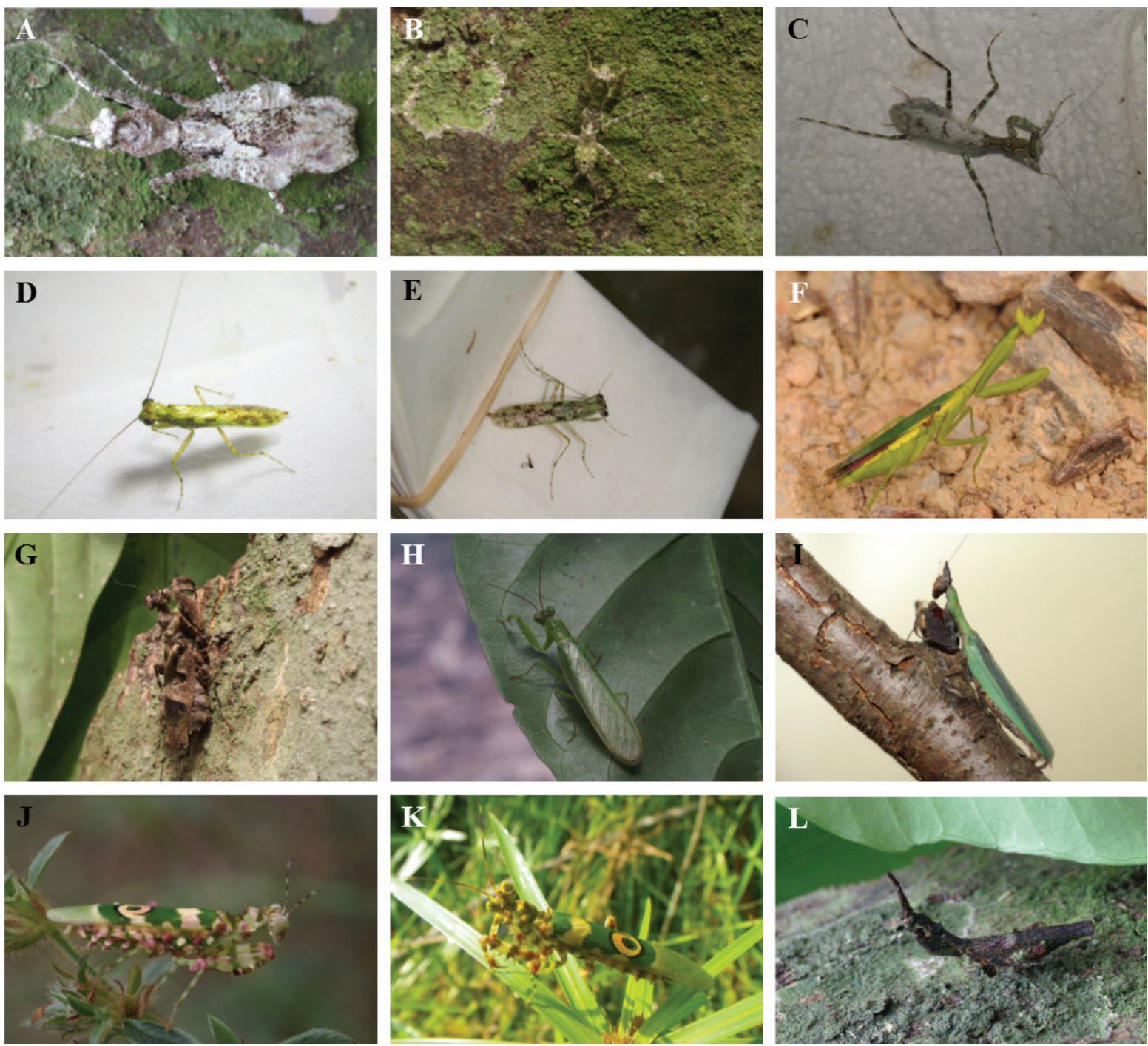

Fig. 4. Live habitus. A. Theopompella aurivillii (female) from DNNP; B. Theopompella sp. (young) from DNNP; C. Dactylopteryx flexuosa (female) from DNNP; D. Amorphoscelis griffinii (male) from DNNP; E. Amorphoscelis pulchra (male) from DNNP; F. Galinthias amoena (female) from LNP; G. Panurgica feae (female) from DNNP; H. Chloroharpax modesta (male) from DNNP; I. Anasigerpes bifasciata (female) from DNNP; J. Pseudocreobotra ocellata (female) from LNP; K. Pseudocreobotra ocellata (male) from DNNP; L. Oxypiloidea (Catasigerpes) margarethae (female) from DNNP.

\section{Results}

Taxonomic checklist. - A total of 1232 specimens (7 families, 36 genera, 1125 males, 89 females, 18 nymphs) were recovered, representing 71 species.

\section{Chroicopteridae}

\section{Genus Entella Stål, 1877}

Note._Entella sp. Unidentified at the moment, research is required about this genus in Congo Basin.

Material examined.-CAR, Dzanga-Sangha Special Reserve, Bayanga, UV trap 15-20.II.1986 (ð̊) (Collector PA) (RCNM).

\section{Amorphoscelidae, Amorphoscelinae Genus Amorphoscelis Stål, 1871}

Amorphoscelis angolica (Beier, 1969)

Beier 1969. Publcoes. Cult. Co. Diam. Angola. 81: 15.

Type locality.-Dundo (Angola).

Material examined._CAR, Dzanga-Ndoki National Park, Lake 3, UV trap 15-23.II.2012 (2へ) (Collector NM and PA) (RCNM); Molongo, border of Sangha River, UV trap 24.I.2012 (ð̋) (Collector NM and PA) (IDM).

Distribution. -Angola, CAR. 


\section{Amorphoscelis griffinii Giglio-Tos, 1913}

Giglio-Tos 1913. Genera Insect. 144: 9.
Amorphoscelis lamottei Roy, 1963

Roy 1963. Mem. Inst. Fond. Afr. Noire 66: 173
Type locality. - "Punta Frailes nell'isola Fernando Po", Bioko Island, Equatorial Guinea.

Material examined._CAR, Dzanga-Ndoki National Park, Lake 1, platform on the canopy, 45m, "Azobé" Lophira alata, Ochnaceae, Genitalia Roy 4404, UV trap 01.XII.2010 (ð) (Collector NM and PA) (RCNM); Buffalos bay, trunk, barcoding BOLD LopeMAN14-236, day capture 26.I.2012 (ㅇ) (Collector NM and PA) (RCNM); Lake 1, UV trap 10.II.2012 (ð) (Collector NM and PA) (RCNM); Lake 1, laboratory tent, night capture 23.II.2012 (ð) (Collector NM and PA) (IDM); Lake 1, platform on the canopy, 45m, "Ayous" Triplochiton scleroxylon, Malvaceae, UV trap 24.II.2012 (ठఓ) (Collector NM and PA) (IDM); Lake 1, trunk of "M'boko" Balanites wilsoniana, Zygophylaceae, day capture tree-climbing 28.II.2012 (ふ) (Collector G. Duvot and E. Le Couillard) (RCNM).

Distribution.-Bioko Island, Cameroon, CAR, Democratic Republic of the Congo, Gabon, Ivory Coast, Rwanda.

\section{Amorphoscelis grisea Bolivar, 1908}

Bolivar 1908b. Dt. Ent. Z. 1908: 515.

Type locality.-Cameroon.

Material examined.-CAR, Komassa, UV trap 05.VIII.1966 (ふ) (Collector M. Boulard) (MNHN); Dzanga-Ndoki National Park, Lidjombo, platform on the canopy, 38m, "Ayous" Triplochiton scleroxylon, Malvaceae, Genitalia Roy 4252, UV trap 25.II.2005 (ð) (Collector PA) (RCNM); Dzanga-Sangha Special Reserve, Bayanga, platform on the canopy 40m, "Kungu" Piptadenastrium africanum, Fabaceae, UV trap 23.X.2008 (ठ) (Collector PA) (IDM); Dzanga-Ndoki National Park, Molongo, Sangha river bank, UV trap 14.XI.2010 (ठ) (Collector NM and PA) (RCNM); Lake 5, clearing in forest, UV trap 29.XI.2010 (ð) (Collector NM and PA) (IDM); Molongo, Sangha river bank, aerial multidirectional trap (PIMUL) 04.XII.2010 (ㅇ) (Collector NM and PA) (RCNM); Dzanga-Sangha Special Reserve, Bayanga, night capture on leaf 23.I.2012 (ð) (Collector NM and PA) (IDM); Dzanga-Ndoki National Park, Lake 1, trunk, barcoding BOLD LopeMAN14-239, night capture 31.I.2012 (young) (Collector NM and PA) (RCNM); Lake 1, trunk, day capture 03.II.2012 (ふ) (Collector NM and PA) (RCNM); Lake 1, UV trap 10.II.2012 (2今) (Collector NM and PA) (IDM); Lake 1, platform on the canopy 38m, "Azobé" Lophira alata, Ochnaceae, UV trap 12.II.2012 (ठ̊) (Collector NM and PA) (IDM); Lake 1, UV trap 13.II.2012 (ठ) (Collector NM and PA) (IDM); Lake 1, laboratory tent, night capture 13.II.2012 (ठ) (Collector NM and PA) (IDM); Lake 3, UV trap 15.II.2012 (ठ̋) (Collector NM and PA) (IDM); Lake 1, leaf, day capture 22.II.2012 (ð) (Collector NM and PA) (IDM); Lake 1, platform on the canopy 45m, "Ayous" Triplochiton scleroxylon, Sterculariaceae, UV trap 22.II.2012 (ठ) (Collector NM and PA) (RCNM); Lake 3, UV trap 22.II.2012 (ठ̊) (Collector NM and PA) (IDM); Lake 1, platform on the canopy $45 \mathrm{~m}$, "Ayous" Triplochiton scleroxylon, Sterculariaceae, barcoding BOLD LopeMAN14-234 $\left(1{ }^{\Uparrow}\right)$, UV trap 24.II.2012 (3今) (Collector NM and PA) (RCNM).

Distribution.-Cameroon, CAR, Congo, Democratic Republic of the Congo, Gabon, Guinea, Ivory Coast, Uganda.
Type locality._-Nimba, Ziela (Guinea).

Material examined.-CAR, Dzanga-Sangha Special Reserve, Bayanga-Lidjombo, PK 21 toward south-west, Genitalia Roy 4247, UV trap 16.VI.1998 (ठ) (Collector PA) (RCNM); Dzanga-Sangha Special Reserve, Bayanga, platform on the canopy 54m, "Ayous" Triplochiton scleroxylon, Sterculariaceae, UV trap 13.X.2008 (20̂) (Collector PA) (IDM); Dzanga-Sangha Special Reserve, Bayanga, platform on the canopy 54m, "Ayous" Triplochiton scleroxylon, Sterculariaceae, UV trap 14.X.2008 (ð̋) (Collector PA) (IDM); Dzanga-Ndoki NP, Lake 1, platform on the canopy 38m, "Azobé" Lophira alata, Ochnaceae, UV trap 12.II.2012 (ठ઼) (Collector NM and PA) (IDM).

Distribution.-CAR, Congo, Democratic Republic of the Congo, Gabon, Ghana, Guinea, Ivory Coast, Tanzania, Uganda.

\section{Amorphoscelis pulchra Bolivar, 1908}

Bolivar 1908. Dt. Ent. Z. 1908: 515.

Type locality.-Cameroon.

Material examined. -CAR, Salo, UV trap 11.IX.1966 (ð) (Collector M. Boulard) (MNHN); Dzanga-Ndoki National Park, Lidjombo, near little bay, UV trap 04-06.II.2005 (4§) (Collector PA) (RCNM and IDM); Dzanga-Ndoki National Park, Lake 1, platform on the canopy 35m, "Azobé" Lophira alata, Ochnaceae, UV trap 22.XI.2010 (20) (Collector NM and PA) (IDM); Dzanga-Ndoki National Park, Lake 1, trunk, day capture 24.XI.2010 (ठ) (Collector NM and PA) (IDM); Dzanga-Ndoki National Park, Lake 1, platform on the canopy 35m, "Azobé" Lophira alata, Ochnaceae, UV trap 25-30.XI.2010 (8ふึ) (Collector NM and PA) (IDM and RCNM); Dzanga-Ndoki National Park, Buffalos bay UV trap 26.I.2012 (J) (Collector NM and PA) (IDM); Dzanga-Ndoki National Park, Lake 1, platform on the canopy 38m, "Azobé" Lophira alata, Ochnaceae, UV trap 05-07.II.2012 (8 $0^{\wedge}$ ) (Collector NM and PA) (IDM and RCNM); Dzanga-Ndoki National Park, Lake 1, trunk, day capture 08.II.2012 (ठ) (Collector NM and PA) (IDM); Dzanga-Ndoki National Park, Lake 1, platform on the canopy 45m, "Ayous" Triplochiton scleroxylon, Sterculariaceae, UV trap 1524.II.2012 (10^) (Collector NM and PA) (IDM).

Distribution.-Cameroon, CAR, Congo, Democratic Republic of the Congo, Gabon, Ghana, Ivory Coast, Sierra Leone.

\section{Amorphoscelis villiersi Roy, 1984}

Roy 1984. Revue fr. Ent. 6(2): 76.

Type locality._Dimonika (Democratic Republic of the Congo).

Material examined.-CAR, Dzanga-Sangha Special Reserve, Bayanga, base of a "Kungu" Piptadenestrium africanum, Fabaceae, Genitalia Roy 4249 and Roy 4255, UV trap 19-23.X.2008 (2 ఓ厃) (Collector PA) (IDM and RCNM).

Distribution. - CAR, Democratic Republic of the Congo. 


\section{Genus Bolivaroscelis Roy, 1973}

\section{Bolivaroscelis bolivarii (Giglio-Tos, 1913)}

Giglio-Tos 1913. Genera Insect. 144: 9.

Type locality.-Johann-Albrechtshöhe (North Cameroon).

Material examined.-CAR, Dzanga-Sangha Special Reserve, Bayanga, platform on the canopy 44m, "Kungu" Piptadenestrium africanum, Fabaceae, Genitalia Roy 4248, UV trap 23.X.2008 (ð̋) (Collector PA) (RCNM).

Distribution.-Cameroon, CAR, Congo, Democratic Republic of the Congo, Gabon.

\section{Genus Caudatoscelis Roy, 1973}

Caudatoscelis caudata Giglio-Tos, 1914

Giglio-Tos 1914 [1913]. Bull. Soc. Ent. Italica 45: 47

Type locality.-Njole (Gabon).

Material examined. - CAR, Dzanga-Ndoki National Park, Lidjombo, in a little bay, UV trap 07.II.2005 () (Collector PA) (RCNM); Lidjombo, trunk, night capture 17.XI.2010 (nymph) (Collector NM and PA) (IDM); Lake 1, trunk, day capture 19.XI.2010 (nymph) (Collector NM and PA) (RCNM); Lake 1, trunk, barcoding BOLD LopeMAN14-233, day capture 30.I.2012 (ㅇ) (Collector NM and PA) (RCNM); Lake 1, UV trap 07.II.2012 (ठ) (Collector NM and PA) (RCNM).

Distribution._CAR, Gabon.

\section{Genus Gigliotoscelis Roy, 1973}

Gigliotoscelis simulans (Giglio-Tos, 1913)

Giglio-Tos 1913. Genera Insect. 144: 10.

Type locality.-Kratoki (Togo).

Material examined._CAR, Dzanga-Ndoki National Park, Lidjombo, platform on the canopy 35m, "Azobé" Lophira alata, Ochnaceae, UV trap 01.II.2005 (ठ) (Collector PA) (IDM); Lidjombo, platform on the canopy 40m, "Kosipo" Entandrophragma candollei, Meliaceae, UV trap 17.II.2005 (ठ̋) (Collector PA) (IDM); Dzanga-Sangha Special Reserve, Bayanga, platform on the canopy 44m, "Kungu" Piptadenastrium africanum, Fabaceae, UV trap 21-23.X.2008 (3 ${ }^{\lambda}$ ) (Collector PA) (IDM and RCNM); Dzanga-Ndoki National Park, Lake 1, platform on the canopy 45m, "Ayous" Triplochiton scleroxylon, Sterculariaceae, UV trap 24.II.2012 (20) (Collector NM and PA) (RCNM).

Distribution._CAR, Cameroon, Congo, Gabon, Ghana, Guinea, Togo.

\section{Genus Maculatoscelis Roy, 1973}

\section{Maculatoscelis ascalaphoides (Bolivar, 1908)}

Bolivar 1908b. Dt. Ent. Z. 1908: 514.
Material examined.-CAR, Dzanga-Sangha Special Reserve, Bayanga, UV trap 15-20.II.1986 (ठ̋) (Collector PA) (IDM); DzangaNdoki NP, Lidjombo, platform on the canopy 35m, "Azobé" Lophira alata, Ochnaceae, UV trap 01.II.2005 (2ð⿱) (Collector PA) (IDM and RCNM); Lidjombo, in a little bay, UV trap 06.II.2005 (ठ) (Collector PA) (IDM); Dzanga-Sangha Special Reserve, Bayanga, platform on the canopy $44 \mathrm{~m}$, "Kungu" Piptadenastrium africanum, Fabaceae, UV trap 23.X.2008 (ð) (Collector PA) (IDM); Dzanga-Ndoki National Park, Lake 1, platform on the canopy 35m, "Azobé" Lophira alata, Ochnaceae, UV trap 21.XI.2010 (ठ) (Collector NM and PA) (RCNM); Lake 5, little clearing in the forest, UV trap 29.XI.2010 (ठ઼) (Collector NM and PA) (IDM); Lake 1 , platform on the canopy 45m, "Azobé" Lophira alata, Ochnaceae, UV trap 29.XI-01.XII.2010 (2た) (Collector NM and PA) (IDM); Molongo, UV trap 24-25.I.2012 (2犬) (Collector NM and PA) (RCNM); Lake 1, platform on the canopy 38m, "Azobé" Lophira alata, Ochnaceae, UV trap 08-12.II.2012 (2仓̂) (Collector NM and PA) (RCNM); Lake 1, platform on the canopy 45m, "Ayous" Triplochiton scleroxylon, Sterculariaceae, UV trap 15.II.2012 (20̂) (Collector NM and PA) (IDM); Lake 3, UV trap 15.II.2012 (ठ઼) (Collector NM and PA) (IDM); Lake 1, base camp, night capture 17.II.2012 (ठఓ) (Collector NM and PA) (IDM); Lake 1, platform on the canopy 45m, "Ayous" Triplochiton scleroxylon, Sterculariaceae, UV trap 24.II.2012 (2今) (Collector NM and PA) (IDM).

Distribution.-Angola, Cameroon, CAR, Congo, Gabon, Ghana, Guinea, Tanzania.

\section{Iridopterygidae, Tropidomantinae, Tropidomantini Genus Negromantis Giglio-Tos, 1915}

Negromantis gracilis (Giglio-Tos, 1915)

Giglio-Tos 1915b. Bull. Soc. Ent. Ital. 46: 52.

Type locality.-Johann-Albrecht Mts (Cameroon).

Material examined.-CAR, Dzanga-Ndoki National Park, Buffalos bay, leaf, barcoding BOLD LopeMAN14-205, day capture 09.II.2012 ( 9 ) (Collector NM and PA) (RCNM); Lake 1, platform on the canopy 38m, "Azobé" Lophira alata, Ochnaceae, barcoding BOLD LopeMAN14-206, UV trap 09.II.2012 (ふ઼) (Collector NM and PA) (RCNM).

Distribution.-Cameroon, CAR.

Negromantis lutescens (Sjöstedt, 1900)

Sjöstedt 1900. Bih. K. svenska VetenskAkad. Handl. 25(6): 15.

Type locality._Kitta (Cameroon).

Material examined.-CAR, Dzanga-Ndoki National Park, Lake 1, leaf, barcoding BOLD LopeMAN14-252, night capture 19.XI.2010

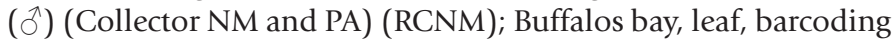
BOLD LopeMAN14-228, night capture 25.I.2012 (q) (Collector NM and PA) (RCNM); Lake 1, Base camp, leaf, barcoding BOLD LopeMAN14-207, night capture 30.I.2012 (+) (Collector NM and PA) (RCNM).

Distribution.-Cameroon, CAR. 


\section{Tarachodidae, Tarachodinae, Tarachodini Genus Galepsus Stål, 1876}

Galepsus (Galepsus) laticeps Werner, 1907

Werner 1907. Sber. Akad. Wiss. Wien 116: 221, 222.

Type locality.-Mukonje, between mundame and Mungo River (Cameroon).

Material examined. - CAR, Dzanga-Sangha Special Reserve, Molongo, Sangha river bank, leaf, night capture 13.XI.2010 (3今) (Collector NM and PA) (RCNM).

Distribution.-Cameroon, CAR, Democratic Republic of the Congo, Gabon, Nigeria.

\section{Galepsus (Galepsus) globiceps Beier, 1942}

Beier 1942. Annln naturh. Mus. Wien 52: 127.

Type locality._Kribi (Cameroon).

Material examined. - CAR, Dzanga-Sangha Special Reserve, Lidjombo, in a little bay, barcoding BOLD LopeMAN14-200 (1今), UV trap 04-10.II.2005 (2乞ै) (Collector PA) (IDM and RCNM).

Distribution. - Cameroon, CAR, Democratic Republic of the Congo.

\section{Galepsus (Syngalepsus) sp.}

Type locality.-Research required and it is the subject of an upcoming revision.

Material examined._CAR, Dzanga-Ndoki National Park, Mboki, Sangha river bank, barcoding BOLD NMMAN11-518 (ठ)), UV trap 24.I.2012 (ð̊) (Collector NM and PA) (RCNM).

\section{Genus Tarachode Burmeister, 1838}

Tarachodes (Tarachodes) pujoli Roy, 2002

Roy 2002. Bull. De la Societe Entomologique de France 107(5): 535.

Type locality._La Maboke (CAR).

Material examined._CAR, Dzanga-Ndoki National Park, Lake 1, base camp, platform on the canopy 38m, "Azobé" Lophira alata, Ochnaceae, Genitalia Moulin NM0068 (1亏) and NM0085 (1ठ), UV trap 06-11.II.2012 (3今) (Collector NM and PA) (IDM and RCNM); Lake 1, base camp, platform on the canopy 45m, "Ayous" Triplochiton scleroxylon, Sterculariaceae, Genitalia Moulin NM0064 (1ठ), UV trap 24.II.2012 (ठ઼) (Collector NM and PA) (RCNM).

Distribution.-CAR.

Tarachodes (Tarachodes) werneri Giglio-Tos, 1911

Giglio-Tos 1911. Bull. Soc. Ital. 42: 113.
Material examined.-CAR, Dzanga-Sangha Special Reserve, Lidjombo, platform on the canopy 35m, "Limba" Terminalia superba, Combretaceae, UV trap 09.II.2005 (ð̋) (Collector PA) (RCNM); Dzanga-Ndoki National Park, Lake 1, base camp, laboratory tent, Genitalia Moulin NM0065, night capture 20.II.2012 (ð) (Collector NM and PA) (RCNM).

Distribution._Cameroon, CAR, Ivory Coast, Ghana, Guinea, Togo.

Tarachodes (Tarachodina) dissimulator Wood-Mason, 1882

Wood-Mason 1882. J. Asiat. Soc. Bengal 51: 23.

Type locality.-Buea Moutains (Cameroon).

Material examined.-CAR, Dzanga-Ndoki National Park, Lake 7, bacording BOLD NMMAN11-521 (1 nymph), day capture 27.XI.2010 (nymph) (Collector NM and PA) (RCNM).

Distribution.-Cameroon, CAR, Gabon, Ghana, Guinea, Ivory Coast, Togo.

Tarachodes (Tarachodina) gerstaeckeri Werner, 1907

Werner 1907. Sber. Akad. Wiss. Wien 116: 215.

Type locality. - Banyaya (Cameroon).

Material examined.-CAR, Komassa, UV trap 05.VIII.1966 (ठ) (Collector M. Boulard) (MNHN); Dzanga-Sangha Special Reserve, between Bayanga and Lidjombo, PK21, UV trap 16.VI.1998 (ठ̂) (Collector PA) (RCNM); Dzanga-Ndoki National Park, Lake 1, base camp, UV trap 28.XI.2010 (ठ̋) (Collector NM and PA) (IDM); Lake 5, in a little clearing, UV trap 29.XI.2010 (ð) (Collector NM and PA) (IDM); Lake 1, base camp, UV trap 07-12.II.2012 (4仓) (Collector NM and PA) (IDM and RCNM); Lake 1, base camp, laboratory tent, Genitalia Moulin NM0067 $(1 \hat{\jmath})$, night capture 14.II.2012 (ð) (Collector NM and PA) (RCNM); Lake 3, Genitalia Moulin NM0066 (10̂), UV trap 18.II.2012 (2犬) (Collector NM and PA) (IDM and RCNM); Lake 1, base camp, UV trap 19.II.2012 (ठ) (Collector NM and PA) (IDM); Mboki, Sangha river bank, trunk, barcoding BOLD NMMAN11-400 (ð̊), day capture 19.II.2012 (ð) (Collector NM and PA) (RCNM); Lake 1, base camp, platform on the canopy 45m, "Ayous", Triplochiton scleroxylon, Sterculariaceae, UV trap 23.II.2012 (ठ) (Collector NM and PA) (IDM); Lake 1, base camp, laboratory tent, night capture 24.II.2012 (§) (Collector NM and PA) (RCNM); Lake 1, base camp, platform on the canopy 45m, "Ayous" Triplochiton scleroxylon, Sterculariaceae, Genitalia Moulin NM0063 (1ठ), UV trap 24.II.2012 (ठ̋) (Collector NM and PA) (RCNM); Lake 3, platform on the canopy 40m, "Sapelli", Entandrophragma cylindricum, Meliaceae, Genitalia Moulin NM0079 (1ठ), UV trap 26.II.2012 (ठ̊) (Collector NM and PA) (RCNM); Lake 1, prospection on "M'Boko" Balanites wilsoniana, Zygophylaceae, trunk, day capture 28.II.2012 (ð) (Collector G. Duvot and E. Le Couillard) (RCNM).

Distribution.-Cameroon, CAR, Ivory Coast, Ghana, Gabon.

Type locality._Misahohe (Togo). 


\section{Miomantidae, Miomantinae, Miomantini} Genus Miomantis Saussure, 1870

Miomantis preussi Karsch, 1892

Karsch 1892b. Ent. Nachr. 18: 148.

Type locality.-Barombi (Cameroon).

Material examined.-CAR, Dzanga-Ndoki National Park, Lake 1, base camp, leaf, night capture 24.XI.2010 (ఓ) (Collector NM and PA) (RCNM); Molongo, Sangha river bank, Genitalia Moulin NM0086, leaf, day capture 24.I.2012 (ठ) (Collector NM and PA) (RCNM); Molongo, trunk, night capture 24.I.2012 (ठ̊) (Collector NM and PA) (IDM); Lake 1, base camp, trunk, night capture 26.I.2012 (q) (Collector NM and PA) (RCNM); Lake 1, base camp, laboratory tent, night capture 01.II.2012 (q) (Collector NM and PA) (RCNM); Lake 1, base camp, leaf, night capture 02.II.2012 (ㅇ) (Collector NM and PA) (IDM); Lake 1, base camp, laboratory tent, night capture 28.II.2012 (ㅇ) (Collector NM and PA) (RCNM).

Distribution. - Bioko Island, Cameroon, CAR, Gabon.

\section{Galinthiadidae Genus Congoharpax La Greca, 1954}

Congoharpax aberrans, La Greca, 1954

La Greca 1954. Annuar. Inst. Mus. Zool. Univ. Napoli 6(2): 38.

Type locality.-Kibalu-Ituri, Mongbwalu, Tshuapa Boende (Democratic Republic of the Congo).

Material examined._CAR, Dzanga-Ndoki National Park, Lidjombo, platform on the canopy 35m, "Azobé" Lophira alata, Ochnaceae, UV trap 01.II.2005 (ð) (Collector PA) (IDM); Dzanga-Sangha Special Reserve, Bayanga, platform on the canopy 44m, "Kungu" Piptadenastrium africanum, Fabaceae, UV trap 22-23.X.2008 (2ङ) (Collector PA) (IDM); Dzanga-Ndoki National Park, Lake 1, clearing in the forest, UV trap 02.XII.2010 (ð) (Collector NM) (RCNM); Lake 1, platform on the canopy 38m, "Azobé" Lophira alata, Ochnaceae, UV trap 04-07.II.2012 (2ふ) (Collector NM and PA) (RCNM); Lake 3, UV trap 16.II.2012 (ठ) (Collector NM and PA) (RCNM); Lake 1, platform on the canopy 45m, "Ayous", Triplochiton scleroxylon, Sterculariaceae, barcoding BOLD NMMAN11-422 (1ठ), Genitalia Moulin NM0046 (1ठ), UV trap 2224.II.2012 (2犬̂) (Collector NM and PA) (RCNM).

Distribution.-Cameroon, CAR, Congo, Democratic Republic of the Congo, Gabon, Ghana, Guinea, Ivory Coast, Togo.

\section{Genus Galinthias Stål, 1877}

Most recent genus revision (Roy and Stiewe 2014).

Galinthias amoena (Saussure, 1871)

Saussure 1871. Mem. Soc. Hist. nat. Geneve 21: 442.

Type locality.-Natal (South Africa).
Material examined._CAR, Dzanga-Ndoki National Park, Lake 1, platform on the canopy 38m, "Azobé" Lophira alata, Ochnaceae, UV trap 06.II.2012 (ふ) (Collector NM) (RCNM); Lake 1, platform on the canopy 38m, "Azobé" Lophira alata, Ochnaceae, UV trap 08.II.2012 (ठ゚) (Collector NM) (RCNM); Lake 1, clearing in the forest, UV trap 09.II.2012 (2犬) (Collector NM) (RCNM); Lake 3, UV trap 22.II.2012 (ठ̂) (Collector NM) (RCNM); Lake 1 , platform on the canopy $45 \mathrm{~m}$, "Ayous" Triplochiton scleroxylon, Sterculariaceae, UV trap 24.II.2012 (ふ઼) (Collector NM) (RCNM).

Distribution.-Botswana, Cameroon, CAR, Congo, Democratic Republic of the Congo, Gabon, Kenya, Malawi, Mozambique, Namibia, Sierra Leone, Somalia, South Africa, Tanzania, Uganda, Zambia, Zimbabwe.

\section{Galinthias occidentalis Beier, 1930}

Beier 1930. Ann. Mag. Nat. Hist. (10) 6: 456.

Type locality._Njala (Sierra Leone).

Material examined.-CAR, Dzanga-Ndoki National Park, Lake 3, UV trap 15.II.2012 (ð) (Collector NM and PA) (RCNM); Lake 1, platform on the canopy 45m, "Ayous" Triplochiton scleroxylon, UV trap 22.II.2012 (ð) (Collector NM and PA) (RCNM).

Distribution.—Cameroon, CAR, Ivory Coast, Gabon, Ghana, Guinea, Sierra Leone.

\section{Hymenopodidae, Acromantinae, Otomantini Genus Chrysomantis Giglio-Tos, 1915}

Most recent genus revision (Roy 2013a).

Chrysomantis cachani Roy, 1964

Roy 1964. Bull. Inst. Fond. Afr. Noire (A) 26: 776.

Type locality._Grabo (Ivory Coast).

Material examined.-CAR, Dzanga-Ndoki National Park, Buffalos bay, UV trap 26.I.2012 (ふ) (Collector NM and PA) (IDM); Lake 1, platform on the canopy 38m, "Azobé" Lophira alata, Ochnaceae, barcoding BOLD NMMAN11-510 (1ठ), UV trap 05-07.II.2012 (2今) (Collector NM and PA) (RCNM); Lake 1, base camp, laboratory tent, night capture 14.II.2012 (つ) (Collector NM and PA) (IDM); Lake 1, leaf, on the canopy, "Moukouloungou" Autramella congolensis, Sapotaceae, night capture 22.II.2012 (ð) (Collector NM and PA) (IDM); Lake 1, base camp, UV trap 22.II.2012 (ठ) (Collector NM and PA) (RCNM); Lake 1, platform on the canopy 45m, "Ayous" Triplochiton scleroxylon, Sterculariaceae, barcoding BOLD NMMAN11-509 (1ठ), UV trap 23.II.2012 (ठ) (Collector NM and PA) (RCNM); Lake 1, day capture on "M'Boko" Balanites wilsoniana, Zygophylaceae, Genitalia Moulin NM0042, trunk, day capture 28.II.2012 (ठ) (Collector G. Duvot and E. Le Couillard) (RCNM).

Distribution._CAR, Congo, Gabon, Ghana, Guinea, Ivory Coast. 
Chrysomantis speciosa Giglio-Tos, 1915

Giglio-Tos 1915. Bull. Soc. Ent. Ital. 46: 103.

Type locality.-Aburi (Ghana).

Material examined.-CAR, Dzanga-Sangha Special Reserve, Bayanga, platform on the canopy $44 \mathrm{~m}$, "Kungu" Piptadenastrium africanum, Fabaceae, UV trap 21-23.X.2008 (4ð) (Collector PA) (IDM); Dzanga-Ndoki National Park, Lake 1, base camp, UV trap 12.II.2012 (ठ) (Collector NM and PA) (IDM); Lake 1, platform on the canopy 45m, "Ayous" Triplochiton scleroxylon, Sterculariaceae, barcoding BOLD NMMAN11-508 (1ठ), UV trap 23-24.II.2012 (20) (Collector NM and PA) (IDM and RCNM); Lake 1, day capture on "M'Boko" Balanites wilsoniana, Zygophylaceae, Genitalia Moulin NM0041, barcoding BOLD NMMAN11-507 (1今), UV trap 28.II.2012 (ठ) (Collector G. Duvot and E. Le Couillard) (RCNM).

Distribution._Angola, CAR, Congo, Gabon, Ghana, Ivory Coast.

\section{Genus Anasigerpes Giglio-Tos, 1915}

Anasigerpes amieti Roy, 1963

Roy 1963. Mem. Inst. Fond. Afr. Noire 66: 199.

Type locality._Nimba Mt, Ziela (Guinea).

Material examined. -CAR, Dzanga-Ndoki National Park, platform on the canopy 45m, "Ayous" Triplochiton scleroxylon, barcoding BOLD NMMAN11-503 (1ठ), UV trap 24.II.2012 (ठ)) (Collector NM and PA) (RCNM); Lake 1, prospection on "M'boko" Balanites wilsoniana, Zygophylaceae, trunk, day capture 28.II.2012 (こ) (Collector G. Duvot and E. Le Couillard) (RCNM).

Distribution.-CAR, Congo, Ghana, Guinea, Ivory Coast, Sierra Leone.

\section{Anasigerpes bifasciata Giglio-Tos, 1915}

Giglio-Tos 1915. Boll. Musei Zool. Anat. Comp. R. univ. Torino 30(702): 14 .

Type locality.-Estudan-Manf, signal Bascho (Cameroon).

Material examined.-CAR, Dzanga-Sangha Special Reserve, Bay-

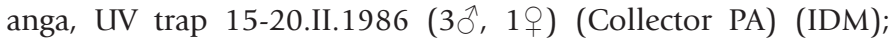
Bayanga, direction Lidjombo PK15, UV trap 06.VI.1998 (ふఓ) (Collector PA) (IDM); Bayanga, direction Lidjombo PK21, UV trap 16.VI.1998 (ô) (Collector PA) (IDM); Lidjombo, platform on the canopy 35m, "Azobé" Lophira alata, Ochnaceae, UV trap 01-02. II.2005 (4仓̃) (Collector PA) (IDM); Lidjombo, in a little Bay, UV trap 04.II.2005 (3ठ) (Collector PA) (IDM); Lidjombo, platform on the canopy 38m, "Ayous" Triplochiton scleroxylon, Sterculariaceae, UV trap 24-25.II.2005 (3 $\overbrace{}^{\lambda})$ (Collector PA) (IDM and RCNM); Bayanga, Sangha river bank, UV trap 09.X.2008 (ð̋) (Collector PA) (IDM); Bayanga, platform on the canopy 54m, "Ayous" Triplochiton scleroxylon, Sterculariceae, UV trap 13-17.X.2008 $(9 \hat{\jmath}, 5$ + ) (Collector PA) (IDM); Bayanga, platform on the canopy 44m, "Kungu" Piptadenastrium africanum, Fabaceae, UV trap 21-23.X.2008 (14 $\hat{\jmath}$, 4 이 (Collector PA) (IDM); Dzanga-Ndoki National Park, Lake 1, laboratory tent, night capture 29-30.XI.2010 (3ㅇ) (Collector NM and PA) (IDM); Lake 1, platform on the canopy 45m, "Azobé" Lophira alata, Ochnaceae, UV trap 30.XI-01.XII.2010 (2ठ) (Collector NM and PA) (IDM); Dzanga-Sangha Special Reserve, Bayanga, Doli Lodge, night capture 04.XII.2010 (ð) (Collector NM and PA) (IDM); Dzanga-Ndoki National Park, Molongo, UV trap 24.I.2012 (ठ) (Collector NM and PA) (RCNM); Lake 1, UV trap 29.I.2012 (4 $\hat{O})$ (Collector NM and PA) (RCNM); Lake 7, platform on the canopy, "Limba" Terminalia superba, Combretaceae, UV trap 03.II.2012 (ठ) (Collector NM and PA) (RCNM); Lake 1, UV trap 04.II.2012 (ㅇ) (Collector NM and PA) (RCNM); Lake 1, platform on the canopy 38m, "Azobé" Lophira alata, Ochnaceae, UV trap 04-07.II.2012 (21§) (Collector NM and PA) (RCNM); Lake 1, Base camp, UV trap 09.II.2012 (ㅇ) (Collector NM and PA) (RCNM); Lake 1, platform on the canopy 38m, "Azobé" Lophira alata, Ochnaceae, UV trap 09.II.2012 (ô) (Collector NM and PA) (RCNM); Lake 1, Base camp, UV trap 10.II.2012 $(5 \hat{\jmath}, 1$ + $)$ (Collector NM and PA) (RCNM); Lake 1, platform on the canopy 38m, "Azobé" Lophira alata, Ochnaceae, UV trap 12.II.2012 $(3 \hat{\jmath})$ (Collector NM and PA) (RCNM); Lake 1, Base camp, UV trap 12.II.2012 $(6 \hat{\circ}, 1+$ ) (Collector NM and PA) (RCNM); Lake 1, platform on the canopy 45m, "Ayous" Triplochiton scleroxylon, Sterculariaceae, UV trap 15.II.2012 (3ڤึ) (Collector NM and PA) (RCNM); Lake 3, UV trap 15.II.2012 (20) (Collector NM and PA) (RCNM); Lake 1, UV trap 19.II.2012 (ठ̊) (Collector NM and PA) (RCNM); Lake 1, laboratory tent, night capture 20.II.2012 (ㅇ) (Collector NM and PA) (RCNM); Lake 1, UV trap 21.II.2012 (5 $\hat{O}^{\text {, }}$ 1 ㅇ) (Collector NM and PA) (RCNM); Lake 1, platform on the canopy 45m, "Ayous" Triplochiton scleroxylon, Sterculariaceae, UV trap 22.II.2012 (3 $\hat{0}, 1$ ㅇ) (Collector NM and PA) (RCNM); Lake 3, UV trap 22.II.2012 (1今, 1) (Collector NM and PA) (RCNM); Lake 1 , platform on the canopy 45m, "Ayous" Triplochiton scleroxylon, Sterculariaceae, UV trap 23-24.II.2012 (11 $\hat{\jmath}, 18$ ) ) (Collector NM and PA) (RCNM); Lake 3, day capture, "Azobé" forest 24.II.2012 (ठ) (Collector NM and PA) (RCNM); Lake 1, platform on the canopy 45m, "Ayous" Triplochiton scleroxylon, Sterculariaceae, UV trap 24.II.2012 $(9 \hat{\delta}, 3$ + ) (Collector NM and PA) (RCNM); Lake 3, platform on the canopy 40m, "Sapelli" Entandrophragma cylindricum, Meliaceae, UV trap 26.II.2012 (ठ̊) (Collector NM and PA) (RCNM); Lake 1, UV trap 26.II.2012 (20, 1) (Collector NM and PA) (RCNM); Lake 7, UV trap 28.II.2012 (O) (Collector NM and PA) (RCNM); Lake 1, day capture on "M'Boko" Balanites wilsoniana, Zygophylaceae, UV trap 28.II.2012 (2+) (Collector G. Duvot and E. Le Couillard) (RCNM).

Distribution.-Angola, Cameroon, CAR, Congo, Democratic Republic of the Congo, Equatorial Guinea, Gabon, Ghana, Guinea, Ivory Coast, Nigeria, Uganda.

\section{Anasigerpes centralis Roy, 1966}

Roy 1966. Bull. Inst. Fond. Afr. Noire A 28: 128.

Type locality._Kivu, Kabunga (Democratic Republic of the Congo).

Material examined.-CAR, Dzanga-Sangha Special Reserve, Bayanga, platform on the canopy 54m, "Ayous" Triplochiton scleroxylon, Sterculariaceae, UV trap 15.X.2008 (ठ̋) (Collector PA) (IDM); Bayanga, platform on the canopy 44m, "Kungu" Piptadenastrium africanum, Fabaceae, UV trap 23.X.2008 (ठ઼) (Collector PA) (IDM); Dzanga-Ndoki National Park, Lake 1, base camp, UV trap 02.XII.2010 (ठ) (Collector NM and PA) (IDM); Lake 1, base camp, 
Genitalia Moulin NM0036, barcoding BOLD NMMAN11-505 (1ठ), UV trap 10.II.2012 (ð) (Collector NM and PA) (RCNM); Lake 1, platform on the canopy 45m, "Ayous" Triplochiton scleroxylon, Sterculariaceae, UV trap 24.II.2012 (1今) (Collector NM and

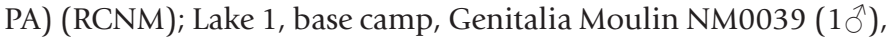
barcoding BOLD NMMAN11-506 (1ठ), UV trap 26.II.2012 (3ð) (Collector NM and PA) (RCNM); Lake 7, night capture 28.II.2012 (ठ) (Collector NM and PA) (RCNM).

Distribution._CAR, Democratic Republic of the Congo.

\section{Anasigerpes grilloti Roy, 1979}

Roy 1979. Bull. Inst. Fond. Afr. Noire 40(1): 92.

Type locality._Brazzaville (Congo).

Material examined._CAR, Dzanga-Ndoki National Park, Lake 1, Base camp, laboratory tent, night capture 29.I.2012 (ð) (Collector NM and PA) (RCNM); Lake 1, Base camp, Genitalia Moulin NM0040 (1今), UV trap 07-12.II.2012 (5今) (Collector NM and PA) (RCNM).

Distribution._CAR, Congo.

\section{Anasigerpes heydeni (Werner, 1908)}

Werner 1908. Ber. Senckenb. Naturf. Ges. 1908: 54.

Type locality.-Unknown.

Material examined.-CAR, Dzanga-Ndoki National Park, Lake 1, platform on the canopy 45m, "Ayous" Triplochiton scleroxylon, Sterculariaceae, barcoding BOLD NMMAN11-504 (1+), UV trap 24.II.2012 (1 +) (Collector NM and PA) (RCNM).

Distribution.-Cameroon, CAR, Congo, Democratic Republic of the Congo, Gabon, Guinea, Ivory Coast, Kenya, Uganda.

\section{Genus Oxypiloidea Schulthess, 1898}

Most recent genus revision (Roy 2013b)

\section{Oxypiloidea (Catasigerpes) centrafricana Roy, 2013}

Roy 2013b. Zoosystema, 35(3): 277-359.

Type locality._La Maboke (Central African Republic).

Material examined._CAR, Dzanga-Ndoki National Park, Lidjombo, platform on the canopy 35m, "Azobé" Lophira alata, Ochnaceae, barcoding BOLD NMMAN11-424 (1ठ), UV trap 02.II.2005 (3ð) (Collector PA) (IDM and RCNM); Lidjombo, in a little bay, UV trap 04-06.II.2005 (3今) (Collector PA) (IDM and RCNM); Dzanga-Sangha Special Reserve, Bayanga, platform on the canopy $44 \mathrm{~m}$, "Kungu" Piptadenastrium africanum, Fabaceae, barcoding BOLD NMMAN11-423 (1ㅇ), UV trap 21-23.X.2008 (1 $\hat{\delta}^{\wedge} ; 1$ 우) (Collector PA) (IDM and RCNM); Ouodo, 90km NE of Nola, UV trap 10-12. VI.2009 (1 $\left.\delta^{\Uparrow}\right)$ (Collector A. Kudrna Jr.) (MSC); Dzanga-Ndoki National Park, Lake 1, laboratory tent, night capture 26-28.XI.2010

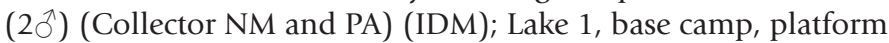

on the canopy 45m, "Azobé" Lophira alata, Ochnaceae, barcoding BOLD LopeMAN14-251 (1ð), UV trap 29-30.XI.2010 (2ð) (Collector NM and PA) (RCNM); Lake 1, platform on the canopy $38 \mathrm{~m}$, "Azobé" Lophira alata, Ochnaceae, UV trap 04-09.II.2012 (3ठㄱ, 1ㅇ) (Collector NM and PA) (IDM and RCNM); Lake 1, base camp, UV trap 12.II.2012 (ð) (Collector NM and PA) (RCNM); Lake 1, platform on the canopy 45m, "Ayous" Triplochiton scleroxylon, Ster-

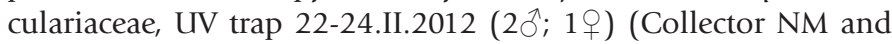
PA) (IDM and RCNM).

Distribution.-Cameroon, CAR, Congo, Democratic Republic of the Congo, Uganda.

\section{Oxypiloidea (Catasigerpes) margarathae (Werner, 1912)}

Werner 1912. Brelin ent. Z. 57: 17.

Type locality.-Dire Daoua (Ethiopia).

Material examined.-CAR, Dzanga-Sangha Special Reserve, Lidjombo, platform on the canopy 38m, "Ayous" Triplochiton scleroxylon, Sterculariaceae, barcoding BOLD NMMAN11-425 (1ठ), UV trap 25.II.2005 (ふ) (Collector PA) (RCNM); Bayanga, platform on the canopy 44m, "Kungu" Piptadenastrium africanum, Fabaceae, UV trap 23.X.2008 (ठ̋) (Collector PA) (RCNM); Dzanga-Ndoki National Park, Lake 1, night capture, Genitalia Moulin NM0037 24.XI.2010 (ठ̋) (Collector S. Danflous) (RCNM); Lake 1, platform on the canopy 38m, "Azobé" Lophira alata, Ochnaceae, barcoding BOLD LopeMAN14-232 ( + ) and -235 (1ठ), UV trap 02-07. II.2012 (1亏; 1 9 ) (Collector NM and PA) (RCNM); Lake 3, UV trap 16.II.2012 (ठ) (Collector NM and PA) (IDM); Lake 1, day capture 21.II.2012 (§) (Collector NM and PA) (IDM); Lake 1, platform on the canopy 45m, "Ayous" Triplochiton scleroxylon, Sterculariaceae, UV trap 23.II.2012 (4仓ે) (Collector NM and PA) (IDM and RCNM).

Distribution.-Cameroon, CAR, Chad, Democratic Republic of the Congo, Eritrea, Ethiopia, Gabon, Kenya, Niger, Nigeria, Sudan, Uganda.

\section{Hymenopodidae, Hymenopodinae, Hymenopodini Genus Chlidonoptera Karsch, 1892}

\section{Chlidonoptera sp.}

Note.-See discussion about this case in the section on DNA barcoding results.

Type locality.-Research required and it is the subject of an upcoming revision.

Material examined._CAR, Dzanga-Sangha Special Reserve, between Bayanga and Lidjombo, PK15, UV trap 31.V.1998 (2ð) (Collector PA) (RCNM); between Bayanga and Lidjombo, PK21, UV trap 02-16.VI.1998 (5今⿱) (Collector PA) (RCNM); Lidjombo, in a little bay, UV trap 05-06.II.2005 (7ठ̋) (Collector PA) (RCNM); Lidjombo, base camp, day capture 08.II.2005 (ð) (Collector PA) (RCNM); Lidjombo, forest, UV trap 13.II.2005 (ð̋) (Collector PA) (RCNM); Bayanga, base camp, leaf, night capture 20.X.2008 (ð) (Collector PA) (RCNM); Bayanga, platform on the canopy $44 \mathrm{~m}$, "Kungu" Piptadenastrium africanum, Fabaceae, UV trap 23.X.2008 (ठ) (Collector PA) (RCNM); camp near a "Kungu" Piptadenastri- 
um africanum, Fabaceae, UV trap 24.X.2008 (3今) (Collector PA) (RCNM); Bayanga, base camp, UV trap 11.X.2008 (2今) (Collector PA) (RCNM); Dzanga-Ndoki National Park, Lake 1, base camp, UV trap 26.XI.2010 (3今) (Collector NM and PA) (RCNM); Lake 1, base camp, laboratory tent, night capture 30.XI-02.XII.2010 (3今) (Collector NM and PA) (RCNM); Lake 5, little clearing in the forest, UV trap 29.XI.2010 (ठ) (Collector NM and PA) (RCNM); Dzanga-Sangha Special Reserve, Bayanga, WWF, wall of WWF guest house, night capture 21.I.2012 (ఓ ) (Collector NM and PA) (RCNM); Dzanga-Ndoki National Park, Mboki, South of Likembe, UV trap 25.I.2012 (2ð) (Collector NM and PA) (RCNM); Lake 1, base camp, barcoding BOLD NMMAN11-405 (1ठ), UV trap 0102.II.2012 (6今) (Collector NM and PA) (RCNM); Lake 7, platform on the canopy, "Limba" Terminalia superba, Combretaceae, UV trap 03.II.2012 (ð) (Collector NM and PA) (RCNM); Lake 1, base camp, barcoding BOLD NMMAN11-404 and LopeMAN14-062, UV trap 04.II.2012 (2亏) (Collector NM and PA) (RCNM); Lake 1, platform on the canopy 38m, "Azobé" Lophira alata, Ochnaceae, UV trap 05.II.2012 (ठ̊) (Collector NM and PA) (RCNM); Lake 1, base camp, UV trap 10-13.II.2012 (5今) (Collector NM and PA) (RCNM); Lake 3, UV trap 15.II.2012 (ठ઼) (Collector NM and PA) (RCNM); Lake 1, base camp, UV trap 19.II.2012 (3 $\left.{ }^{\AA}\right)$ (Collector NM and PA) (RCNM); Lake 1, base camp, laboratory tent, night capture 20.II.2012 (ð) (Collector NM and PA) (RCNM); Lake 1, platform on the canopy 45m, "Ayous" Triplochiton scleroxylon, Sterculariaceae, Genitalia Moulin 0069 (1ठ̂), UV trap 22-24.II.2012 (2今) (Collector NM and PA) (RCNM); Lake 3, UV trap 22.II.2012 (ふ) (Collector NM and PA) (RCNM); Lake 7, UV trap 29.II.2012 (ठ) (Collector NM and PA) (RCNM); Lake 1, laboratory tent, barcoding BOLD NMMAN11-398, night capture 02.III.2012 (ð) (Collector NM and PA) (RCNM).

Distribution.-CAR.

\section{Genus Chloroharpax Werner, 1908}

\section{Chloroharpax modesta (Gerstaecker, 1883)}

Gerstaecker 1883. Mitt. Naturw. Ver. Neu-Vorpomm. 14: 82.

Type locality.-Cameroon.

Material examined.-CAR, Dzanga-Sangha Special Reserve, between Bayanga and Lidjombo, PK21, UV trap 15.VI.1998 (ふ) (Collector PA) (IDM); Ouodo, before cross-road between Nola and Berberati, UV trap 20.I.2005 (ठ̋) (Collector PA) (RCNM); Lidjombo, in a little bay, UV trap 04-06.II.2005 (3今) (Collector PA) (IDM); Lidjombo, platform on the canopy 38m, "Ayous" Triplochiton scleroxylon, Sterculariaceae, UV trap 25.II.2005 (ठ) (Collector PA) (IDM); Bayanga, at the basis of "Ayous" Triplochiton scleroxylon, Sterculariaceae, UV trap 18.X.2008 (ð̋) (Collector PA) (IDM); Bayanga, platform on the canopy 44m, "Kungu" Piptadenastrium africanum, Fabaceae, UV trap 23.X.2008 (6今) (Collector PA) (IDM and RCNM); Dzanga-Ndoki National Park, Lake 1, platform on the canopy 45m, "Azobé" Lophira alata, Ochnaceae, UV trap 21.XI.2010 (ठ઼) (Collector NM and PA) (IDM); Lake 1, platform on the canopy 45m, "Azobé" Lophira alata, Ochnaceae, UV trap 2430.XI.2010 (7ठ̂) (Collector NM and PA) (IDM); Dzanga-Sangha Special Reserve, Bayanga, WWF, wall of WWF guest house, night capture 21.I.2012 (ठ) (Collector NM and PA) (RCNM); DzangaNdoki National Park, Lake 1, base camp, UV trap 30.I.2012 (3ð)
(Collector NM and PA) (IDM and RCNM); Lake 1, base camp, UV trap 31.I.2012 (đ) (Collector NM and PA) (RCNM); Lake 1, platform on the canopy 38m, "Azobé" Lophira alata, Ochnaceae, UV trap 04-06.II.2012 (4ठ) (Collector NM and PA) (RCNM); Lake 1, base camp, UV trap 10-12.II.2012 (5今) (Collector NM and PA) (RCNM); Lake 1, platform on the canopy 45m, "Ayous" Triplochiton scleroxylon, Sterculariaceae, UV trap 15.II.2012 ( $\left.{ }^{\lambda}\right)$ (Collector NM and PA) (RCNM); Lake 3, barcoding BOLD NMMAN11-413 and -414, UV trap 18.II.2012 (2仓) (Collector NM and PA) (RCNM); Lake 1, platform on the canopy $45 \mathrm{~m}$, "Ayous" Triplochiton scleroxylon, Sterculariaceae, barcoding BOLD NMMAN11-452 (1ळ), UV trap 22-24.II.2012 (3 ${ }^{\Uparrow}$ ) (Collector NM and PA) (RCNM); Lake 3, platform on the canopy 40m, "Sapelli" Entandophragma cylindricum, Meliaceae, barcoding BOLD NMMAN11-437 (1ठ)), UV trap 26.II.2012 (3今) (Collector NM and PA) (RCNM).

Distribution.-Cameroon, CAR, Congo, Democratic Republic of the Congo, Gabon, Ghana, Guinea, Ivory Coast, Nigeria.

\section{Genus Panurgica Karsch, 1896}

Panurgica feae Griffini, 1907

Griffini 1907. Annali Mus. Civ. Stor. Nat. Genova 43: 414.

Type locality.-Fernando Po Island = Bioko Island (Equatorial Guinea).

Material examined.-CAR, Dzanga-Sangha Special Reserve, between Bayanga and Lidjombo, PK21, UV trap 09-15.VI.1998 (3仓) (Collector PA) (IDM); Lidjombo, in a little bay, UV trap 07.II.2005 (ठ) (Collector PA) (IDM); Lidjombo, platform on the canopy 35m, "Limba" Terminalia superba, Combretaceae, UV trap 09.II.2005 (3 ) (Collector PA) (IDM); Bayanga, camp near a "Kungu" Piptadenastrium africanum, Fabaceae, UV trap 24.X.2008 (ठ) (Collector PA) (IDM); Dzanga-Ndoki National Park, Lake 5, little clearing in the forest, UV trap 29.XI.2010 (ठ)) (Collector NM and PA) (IDM); Lake 1, clearing in the forest, UV trap 02.XII.2010 (ठ̊) (Collector NM and PA) (IDM); Lake 7, barcoding BOLD LopeMAN14-197 (1ठ), UV trap 02.II.2012 (2ð) (Collector NM and PA) (RCNM); Lake 6, day capture 03.II.2012 (ㅇ) (Collector NM and PA) (RCNM); Lake 1, day capture 12.II.2012 (ㅇ) (Collector NM and PA) (RCNM); Lake 1, base camp, UV trap 12.II.2012 (2§) (Collector NM and PA) (RCNM); Lake 1, base camp, laboratory tent, night capture 14.II.2012 (q) (Collector NM and PA) (RCNM); Lake 7, barcoding BOLD LopeMAN14-230, UV trap 28.II.2012 () (Collector NM and PA) (RCNM); Lake 7, leaf, barcoding BOLD LopeMAN14-231 (1ㅇ), day capture 03.III.2012 (2+) (Collector NM and PA) (RCNM).

Distribution.-Angola, Bioko Island, CAR, Equatorial Guinea, Gabon.

\section{Panurgica rehni La Greca, 1954}

La Greca 1954. Annuar. Inst. Mus. Zool. Univ. Napoli. 6(2): 6.

Type locality.-Tshuapa, Eala (Demcratic Republic of the Congo).

Material examined.-CAR, Dzanga-Sangha Special Reserve, Bayanga, UV trap 11.X.2008 (ㅇ) (Collector PA) (RCNM); Bayanga, camp near a "Kungu" Piptadenastrium africanum, Fabaceae, UV 
trap 19.X.2008 (ふ) (Collector PA) (RCNM); Bayanga, platform on the canopy 44m, "Kungu" Piptadenastrium africanum, Fabaceae, UV trap 23.X.2008 (3今) (Collector PA) (IDM); Lake 1, platform on the canopy 38m, "Azobé" Lophira alata, Ochnaceae, barcoding BOLD LopeMAN14-202 and -203 (20), UV trap 04.II.2012 $(3 \hat{\jmath})$ (Collector NM and PA) (RCNM); Lake 1, base camp, laboratory tent, night capture 10.II.2012 (ठ) (Collector NM and PA) (RCNM); Lake 1, base camp, UV trap 10.II.2012 (ð) (Collector NM and PA) RCNM); Lake 1, platform on the canopy 38m, "Azobé" Lophira alata, Ochnaceae, UV trap 11.II.2012 (क) (Collector NM and PA) (RCNM); Lake 1, UV trap 13.II.2012 (ð) (Collector NM and PA) (RCNM); Lake 3, barcoding BOLD NMMAN11-454, UV trap 15.II.2012 (ð) (Collector NM and PA) (RCNM); Lake 1, laboratory tent, night capture 20.II.2012 (ठ゚) (Collector NM and PA) (RCNM); Lake 3, barcoding BOLD NMMAN11-500, UV trap 22.II.2012 (ठ) (Collector NM and PA) (RCNM); Lake 1, platform on the canopy 45m, "Ayous" Triplochiton scleroxylon, Sterculariaceae, barcoding BOLD NMMAN11-399 (1ठ) and -429 (1ठ), UV trap 23-24.II.2012 (3ðै) (Collector NM and PA) (RCNM); Lake 1, day capture on "M'Boko" Balanites wilsoniana, Zygophylaceae, Genitalia Moulin NM0044 and NM0045, trunk, day capture 28.II.2012 $\left(2{ }^{\Uparrow}\right)$ (Collector G. Duvot and E. Le Couillard) (RCNM).

Distribution._CAR, Democratic Republic of the Congo, Gabon.

\section{Genus Pseudocreobotra Saussure, 1870}

\section{Pseudocreobtra ocellata Beauvois, 1805}

Palisot de Beauvois 1805. Ins. Afr. Amer. Orth. p. 110.

Type locality.-Oware (Angola).

Material examined._CAR, Dzanga-Sangha Special Reserve, Bayanga, UV trap 15-20.II.1086 (ふ) (Collector PA) (IDM); DzangaNdoki National Park, Lake 1, Cyperaceae, day capture 20.XI.2010 (2今) (Collector NM and PA) (IDM); Lake 2, Cyperaceae, day capture 21-30.XI.2010 (1 $\hat{0}, 2$ ㅇ) (Collector NM and PA) (IDM); Dzanga-Sangha Special Reserve, Bayanga, airfield, day capture 23.I.2012 (nymph) (Collector NM and PA) (RCNM); DzangaNdoki National Park, Lake 1, Cyperaceae, day capture 02-11. II.2012 (3 nymph, 1 ) (Collector NM and PA) (RCNM); Lake 1, laboratory tent, night capture 13.II.2012 (ठ̊) (Collector NM and PA) (RCNM).

Distribution._Angola, Burkina Faso, Cameroon, CAR, Congo, Democratic Republic of the Congo, Gabon, Ghana, Guinea, Kenya, Liberia, Mozambique, Nigeria, Senegal, Sierra Leone, Tanzania, Togo, Uganda.

\section{Hymenopodidae, Phyllocraniinae Genus Phyllocrania Burmeister, 1838}

\section{Phyllocrania paradoxa Burmeister, 1838}

Burmeister 1838. Handb. Ent., 2: 549.

Type locality.-Cape Province (South Africa).

Material examined.-CAR, Dzanga-Sangha Special Reserve, Bayanga, UV trap 15-20.II.1986 (ठ̊) (Collector PA) (IDM); Lidjombo, in a little bay, UV trap 06.II.2005 (3ð) (Collector PA) (IDM); Lidjombo, near "Oualé" river, barcoding BOLD NMMAN11-499, UV trap 06.II.2005 (ठ̂) (Collector PA) (RCNM); Lidjombo, in a little bay, UV trap 07.II.2005 (2ઈ) (Collector PA) (IDM and RCNM); Dzanga-Ndoki National Park, Molongo, Sangha river bank, UV trap 25.I.2012 (ð) (Collector NM and PA) (RCNM).

Distribution.-Angola, Cameroon, CAR, Congo, Democratic Republic of the Congo, Ethiopia, Ghana, Guinea, Ivory Coast, Kenya, Madagascar?, Mozambique, Namibia, Somalia, South Africa, Sudan, Tanzania, Togo, Uganda, Zimbabwe.

\section{Hymenopodidae, Oxypilinae Genus Junodia Schulthess-Rechberg, 1899}

Junodia lameyi Beier, 1942

Beier 1942. Annln. Naturh. Mus. Wien. 52: 150.

Type locality.-Kribi (Cameroon).

Material examined._CAR, Dzanga-Sangha Special Reserve, Bayanga, Sangha river bank, UV trap 25.X.2008 (ð) (Collector PA) (IDM); Dzanga-Ndoki National Park, Lake 1, platform on the canopy 38m, "Azobé" Lophira alata, Ochnaceae, UV trap 07.II.2012 (ð) (Collector NM and PA) (RCNM).

Distribution.-Angola, Cameroon, CAR, Congo, Gabon, Ghana, Guinea, Ivory Coast.

\section{Hymenopodidae, Sibyllinae Genus Presibylla Bolivar, 1908}

\section{Presibylla speciosa Roy, 1996}

Roy 1996. Bulletin du Museum National d'Histoire Naturelle Section A Zoologie Biologie et Ecologie Animales 18(1-2): 84.

Type locality.-Sapele (Nigeria).

Material examined._CAR, Dzanga-Ndoki National Park, Lake 1, Base camp, platform on the canopy 35m, "Azobé" Lophira alata, Ochnaceae, UV trap 24.XI.2010 (ठ̃) (Collector NM and PA) (IDM); Lake 7, UV trap 28.XI.2010 (2犬) (Collector NM and PA) (IDM); Lake 1, base camp, platform on the canopy 45m, "Azobé" Lophira alata, Ochnaceae, UV trap 29.XI.2010 (ð) (Collector NM and PA) (IDM); Lake 1, base camp, UV trap 01.XII.2010 (ठ) (Collector NM and PA) (IDM); Lake 1, base camp, barco-

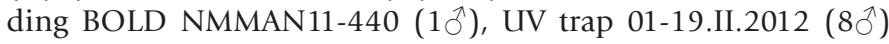
(Collector NM and PA) (RCNM); Lake 1, base camp, laboratory tent, barcoding BOLD NMMAN11-412 (1今̄), night capture 1319.II.2012 (4 $\overbrace{}^{\lambda}$ ) (Collector NM and PA) (RCNM); Lake 1, base camp, platform on the canopy $45 \mathrm{~m}$, "Ayous" Triplochiton scleroxylon, Sterculariaceae, Genitalia Moulin NM0084 (10), barcoding BOLD NMMAN11-456 (1§), UV trap 24.II.2012 (4§) (Collector NM and PA) (RCNM); Lake 3, platform on the canopy 40m, "Sapelli" Entandrophragma cylindricum, Meliaceae, barcoding BOLD NMMAN11-428 (1ठ), UV trap 26.II.2012 (ठ) ) (Collector NM and PA) (RCNM).

Distribution._Cameroon, CAR, Nigeria. 


\section{Genus Sibylla Stål, 1856}

\section{Sibylla (Sibyllopsis) griffinii Giglio-Tos, 1915}

Giglio-Tos 1915b. Bull. Soc. Ent. Ital. 46: 92.

Type locality.-Bioko Island (Equatorial Guinea).

Material examined. - CAR, Dzanga-Sangha Special Reserve, Lidjombo, in forest near base camp, UV trap 27.II.2005 ( $ٌ)(C o l-$ lector PA) (IDM); Bayanga, platform on the canopy $44 \mathrm{~m}$, "Kungu" Piptadenastrium africanum, Fabaceae, UV trap 23.X.2008 (ð) (Collector PA) (IDM); Dzanga-Ndoki National Park, Lake 1, base camp, platform on the canopy 35m, "Azobé" Lophira alata, Ochnaceae, UV trap 25.XI.2010 (ठ) (Collector NM and PA) (IDM); Lake 5, little clearing in the forest, UV trap 29.XI.2010 (ठ) (Collector NM and PA) (IDM); Lake 1, base camp, platform on the canopy 45m, "Azobé" Lophira alata, Ochnaceae, UV trap 01.XII.2010 (ठ̊) (Collector NM and PA) (IDM); Lake 1, base camp, platform on the canopy 38m, "Azobé" Lophira alata, Ochnaceae, Genitalia Moulin NM0052 (1§), UV trap 0510.II.2012 (3ß) (Collector NM and PA) (RCNM); Lake 1, base camp, UV trap 10-12.II.2012 (3今) (Collector NM and PA) (IDM and RCNM); Lake 1, base camp, laboratory tent, night capture 19.II.2012 (ठ) (Collector NM and PA) (RCNM); Lake 1, prospection on "M'boko" Balanites wilsoniana, Zygophylaceae, trunk, day capture 28.II.2012 (q) (Collector G. Duvot and E. Le Couillard) (RCNM).

Distribution.-Benin, Bioko Island, Cameroon, CAR, Gabon, Ghana, Ivory Coast, Liberia, Nigeria, Sierra Leone, Togo.

Sibylla (Sibyllopsis) pannulata Karsch, 1894

Karsch 1894. Berl. Ent. Z. 34(2): 277.

Type locality.-Buea Montains (Cameroon).

Material examined.-CAR, Dzanga-Sangha Special Reserve, between Bayanga and Lidjombo, PK15, UV trap 31.V.1998 (ठ̋) (Collector PA) (RCNM); Between Bayanga and Lidjombo, PK21, UV trap 09-15.VI.1998 (3ठ) (Collector PA) (IDM); Lidjombo, in a little bay, UV trap 05-06.II.2005 (2仓) (Collector PA) (IDM); Bayanga, base camp, UV trap 16.X.2008 (ð) (Collector PA) (IDM); Dzanga-Ndoki National Park, Lake 1, base camp, laboratory tent, night capture 02.XII.2010 (2ð) (Collector NM and PA) (IDM); M'Boki, Sangha river bank, UV trap 25.I.2012 (ठ઼) (Collector NM and PA) (RCNM); Lake 1, platform on the canopy 38m, "Azobé" Lophira alata, Ochnaceae, Genitalia Moulin NM0082 (1ठ), UV trap 04-12.II.2012 (6今; 2ᄋ) (Collector NM and PA) (IDM and RCNM); Lake 1, base camp, UV trap 10-12.II.2012 (2ઈ) (Collector NM and PA) (RCNM); Lake 1, base camp, laboratory tent, barcoding BOLD NMMAN11-457 (1乞̂), night capture 13-17.II.2012 (5今) (Collector NM and PA) (RCNM); Lake 1, base camp, UV trap 19.II.2012 (ठ) (Collector NM and PA) (RCNM); Lake 1, platform on the canopy 45m, "Ayous" Triplochiton scleroxylon, Sterculariaceae, barcoding BOLD NMMAN11-453 (1今), UV trap 24.II.2012 (4仓) (Collector NM and PA) (RCNM).

Distribution.-Angola, Cameroon, CAR, Congo, Democratic Republic of the Congo, Equatorial Guinea, Gabon, Nigeria.
Sibylla (Sibyllopsis) vanderplaetseni Roy, 1963

Roy 1963. Mem. Inst. Fond. Afr. Noire 66: 195.

Type locality._Mount Nimba, Ziela (Guinea).

Material examined._CAR, Dzanga-Sangha Special Reserve, between Bayanga and Lidjombo, PK21, UV trap 09.VI.1998 (2§) (Collector PA) (IDM); Lidjombo, in a little bay near base camp, UV trap 05.II.2005 (ठ) (Collector PA) (IDM); Dzanga-Ndoki National Park, Lake 1, base camp, laboratory tent, night capture 28.XI.2010 (§) (Collector NM and PA) (IDM); Lake 1, base camp, platform on the canopy 45m, "Azobé" Lophira alata, Ochnaceae, UV trap 01.XII.2010 () (Collector NM and PA) (IDM); Lake 1, base camp, Genitalia Moulin NM0072 (1今), UV trap 02-12. II.2012 (3^) (Collector NM and PA) (RCNM).

Distribution.-Angola, Cameroon, CAR, Congo, Democratic Republic of the Congo, Gabon, Ghana, Guinea, Ivory Coast, Uganda.

\section{Deroplatyidae Genus Macrodanuria Sjöstedt, 1900}

\section{Macrodanuria elongata (Borre de Preudhomme, 1883)}

Borre de Preudhomme 1883. Liste Mant. Mus. Bruxelles 76.

Type locality.-Conde (Guinea).

Material examined.-CAR, Dzanga-Ndoki National Park, Lake 1, tent, night capture 29.I.2012 (ठ̊) (Collector NM and PA) (RCNM).

Distribution.-Cameroon, CAR, Gabon, Ghana, Guinea, Ivory Coast.

\section{Genus Dactylopteryx Karsch, 1892}

Dactylopteryx flexuosa Karsch, 1892

Karsch 1892a. Ent. Nachr. 18(1): 8.

Type locality.-Unknown (Gabon).

Material examined.-CAR, Dzanga-Ndoki National Park, Lake 1, trunk, night capture 25-26.XI.2010 (1 nymph; 19) (Collector NM and PA) (RCNM).

Distribution.-Cameroon, CAR, Congo, Gabon, Ghana, Ivory Coast.

Genus Theopompella Giglio-Tos, 1917

Theopompella aurivillii (Sjöstedt, 1900)

Sjöstedt 1900. Bih. K. svenska VetenskAkad. Handl. 25(6): 3.

Type locality.-Mapanga (Cameroon).

Material examined._CAR, Dzanga-Sangha Special Reserve, between Bayanga and Lidjombo, PK15, UV trap 31.V.1998 (ð) (Collector PA) (RCNM); Lidjombo, platform on the canopy $35 \mathrm{~m}$, 
"Limba" Terminalia superba, Combretaceae, UV trap 09.II.2005 (ठ) (Collector PA) (IDM); Bayanga, platform on the canopy $54 \mathrm{~m}$, "Ayous" Triplochiton scleroxylon, Sterculariaceae, UV trap 17.X.2008 (ठ) (Collector PA) (IDM); Bayanga, platform on the canopy 44m, "Kungu" Piptadenastrium africanum, Fabaceae, UV trap 21.X.2008 (2犬) (Collector PA) (IDM and RCNM); Bayanga, base camp, laboratory tent, night capture 21.X.2008 (§) (Collector PA) (IDM); Bayanga, Sangha river bank, UV trap 25.X.2008 (ふ) (Collector PA) (IDM); Dzanga-Ndoki National Park, Lake 1, platform on the canopy 45m, "Azobé" Lophira alata, Ochnaceae, UV trap 01.XII.2010 (ठ) (Collector NM and PA) (IDM); Lake 1, Base camp, laboratory tent, night capture 02.XII.2010 (ô) (Collector NM and PA) (RCNM); Lake 1, base camp, trunk, night capture 08.II.2012 () (Collector PA and NM) RCNM); Buffalos bay, leaf, barcoding BOLD NMMAN11-439, night capture 19.II.2012 (さ) (Collector NM and PA) (RCNM); Lake 1, platform on the canopy 45m, "Ayous" Triplochiton scleroxylon, Sterculariaceae, barcoding BOLD NMMAN11-416, UV trap 24.II.2012 (20⿱) (Collector NM and PA) (RCNM); Lake 7, barcoding BOLD NMMAN11-430, UV trap 29.II.2012 (ठ̊) (Collector NM and PA) (RCNM).

Distribution.-Angola, Bioko Island, Cameroon, CAR, Congo, Democratic Republic of the Congo, Equatorial Guinea, Gabon, Malawi.

\section{Theopompella heterochroa (Gerstaecker, 1883)}

Gerstaecker 1883. Mitt. Ver. Vorpomm. xiv: 80.

Type locality.-Quaqua (Cameroon).

Material examined.-CAR, Dzanga-Sangha Special Reserve, between Bayanga and Lidjombo, PK21, UV trap 13.VI.1998 (ठ̊) (Collector PA) (IDM); Lidjombo, Sangha river bank, UV trap 03.II.2005 (ठ̋) (Collector PA) (IDM); Lidjombo, in forest, UV trap 12-13.II.2005 (2乞) (Collector PA) (IDM); Bayanga, leaf, day capture 18.X.2008 (ㅇ) (Collector PA) (IDM); Bayanga, Sangha river bank, UV trap 25.X.2008 (ð) (Collector PA) (IDM); DzangaNdoki National Park, Lake 1, base camp, UV trap 27.I-10.II.2012 $(4 \hat{\bigcirc})$ (Collector NM and PA) (RCNM); Lake 1, platform on the canopy 45m, "Ayous" Triplochiton scleroxylon, Sterculariaceae, Genitalia Moulin NM0092, UV trap 15.II.2012 (ô) (Collector NM and PA) (RCNM); Dzanga-Sangha Special Reserve, Bayanga, Doli Lodge, leaf, night capture, barcoding BOLD NMMAN11-402, 16.II.2012 (ठ̊) (Collector J.-P. Maurel) (RCNM); Dzanga-Ndoki National Park, Lake 1, laboratory tent, night capture 20.II.2012 (ठ) (Collector NM and PA) (RCNM); Lake 1, leaf, Genitalia Moulin NM0077, 24.II.2012 (1ठ) day capture 23-24.II.2012 (1ठ; 1우) (Collector NM and PA) (RCNM); Lake 1, platform on the canopy 45m, "Ayous" Triplochiton scleroxylon, Sterculariaceae, barcoding BOLD NMMAN11-415, UV trap 24.II.2012 (ठ̊) (Collector NM and PA) (RCNM).

Distribution.-Cameroon, CAR, Democratic Republic of the Congo, Gabon, Kenya, Nigeria.

\section{Theopompella pallida Giglio-Tos, 1916}

Giglio-Tos 1916. Bull. Soc. Ent. Ital. 48: 87.

Type locality.-Galli-Koko (Democratic Republic of the Congo).
Material examined. - CAR, Dzanga-Sangha Special Reserve, Bayanga, platform on the canopy $44 \mathrm{~m}$, "Kungu" Piptadenastrium africanum, Fabaceae, UV trap 23.X.2008 (ठ̋) (Collector PA) (RCNM); Dzanga-Ndoki National Park, Lake 1, base camp, Genitalia NM0087, day capture 31.I.2012 (ð) (NM and PA) (RCNM).

Distribution.-Cameroon, CAR, Democratic Republic of the Congo, Gabon.

\section{Genus Stenopyga Karsch, 1892}

\section{Stenopyga (Stenopyga) ipassa Roy, 1973}

Roy 1973. Biologia gabon. 8(3-4): 256.

Type locality._Plateau Ipassa (Gabon).

Material examined._CAR, Komassa, UV trap 07.VIII.1966 (ठ઼) (Collector M. Boulard) (MNHN); Dzanga-Sangha Special Reserve, between Bayanga and Lidjombo, PK15, UV trap 31.V.1998 $\left(2{ }^{\Uparrow}\right)$ (Collector PA) (IDM); Lidjombo, in a little bay, UV trap 05-10.II.2005 (2ð) (Collector PA) (IDM); Lidjombo, Forest, UV trap 13.II.2005 (ठ) (Collector PA) (IDM); Bayanga, base camp, Tent, night capture 18-19.X.2008 (2た̂) (Collector PA) (IDM); Bayanga, platform on the canopy trap 44m, "Kungu" Piptadenastrium africanum, Fabaceae, UV trap 23.X.2008 ( ( $\left.{ }^{\star}\right)$ (Collector PA) (IDM); Bayanga, base camp, Tent, night capture 24.X.3008 (ठ̂) (Collector PA) (IDM); Bayanga, Sangha river bank, UV trap 25.X.2008 (ठ઼) (Collector PA) (IDM); Dzanga-Ndoki National Park, Lake 7, UV trap 28.XI.2010 (ठ̂) (Collector NM and PA) (IDM); Lake 1, base camp, laboratory tent, night capture 29.XI.2010 ( $\lesssim$ ) (Collector NM and PA) (IDM); Dzanga-Sangha Special Reserve, Bayanga, Town, leaf, night capture 23.I.2012 (ठ) (Collector NM and PA) (RCNM); Dzanga-Ndoki National Park, M'Boki, Sangha river bank, UV trap 25.I.2012 (ठ̋) (Collector NM and PA) (RCNM); Lake 1, base camp, trunk, night capture 26.I.2012 (ठ̃) (Collector NM and PA) (RCNM); Lake 1, base camp, UV trap 27.I.2012 (ठ) (Collector NM and PA) (RCNM); Lake 1, base camp, Remote Canopy Trap, UV trap 28.I.2012 ( $\lesssim$ (Collector NM and PA) (IDM); Lake 1, base camp, UV trap 29.I-02.II.2012 (4ठ) (Collector NM and PA) (IDM and RCNM); Lake 7, UV trap 03.II.2012 (đ̋) (Collector NM and PA) (RCNM); Lake 1, base camp, UV trap 07.II.2012 (20) (Collector NM and PA) (RCNM); Likembe, Sangha river bank, UV trap 09.II.2012 (ठ) (Collector NM and PA) (RCNM); Lake 1, base camp, UV trap 10.II.2012 (2犬) (Collector NM and PA) (RCNM); Lake 1, base camp, laboratory tent, night capture 11.II.2012 (ð) (Collector NM and PA) (RCNM); Lake 1, base camp, UV trap 1213.II.2012 (2犬) (Collector NM and PA) (RCNM); Lake 1, base camp, laboratory tent, barcoding BOLD NMMAN11-409, night capture 14.II.2012 (ठ)) (Collector NM and PA) (RCNM); Lake 1, platform on the canopy 45m, "Ayous" Triplochiton scleroxylon, Sterculariaceae, UV trap 15.II.2012 (20) (Collector NM and PA) (RCNM); Lake 3, UV trap 15-16.II.2012 (2犬) (Collector NM and PA) (RCNM); Lake 1, base camp, laboratory tent, barcoding BOLD NMMAN11-446 (1ठ), night capture 17-19.II.2012 (2ठ) (Collector NM and PA) (RCNM); Lake 1, base camp, day capture 22.II.2012 (ठ) (Collector NM and PA) (RCNM); Lake 1, base camp, night capture 22.II.2012 (ð) (Collector NM and PA) (IDM); Lake 3, barcoding BOLD NMMAN11-411 and -445 (2ð) 
UV trap 22.II.2012 (3ठ) (Collector NM and PA) (RCNM); Lake 1 , platform on the canopy $45 \mathrm{~m}$, "Ayous" Triplochiton scleroxylon, Sterculariacae, UV trap 24.II.2012 (ふ) (Collector NM and PA) (RCNM); Lake 3, platform on the canopy 40m, "Sapelli" Entandrophragma cylindricum, Meliaceae, barcoding BOLD NMMAN11-434, UV trap 26.II.2012 (ð̋) (Collector NM and PA) (RCNM); Lake 1, base camp, UV trap 26.II.2012 (ð) (Collector NM and PA) (RCNM).

Distribution._CAR, Gabon.

\section{Stenopyga (Stenopyga) ziela Roy, 1963}

Roy 1963. Mem. Inst. fond. Afr. noire 66: 183.

Type locality.-Mount Nimba, Ziela (Guinea).

Material examined.-CAR, Dzanga-Sangha Special Reserve, between Bayanga and Lidjombo, PK21, UV trap 15-16.VI.1998 (2ङ) (Collector PA) (IDM); Lidjombo, platform on the canopy $35 \mathrm{~m}$, "Limba" Terminalia superba, Combretaceae, UV trap 09.II.2005 (ð) (Collector PA) (IDM); Bayanga, base camp, tent, night capture 21.X.2008 (ठ) (Collector PA) (IDM); Bayanga, platform on the canopy 44m, "Kungu" Piptadenastrium africanum, Fabaceae, UV trap 23.X.2008 (2犬) (Collector PA) (IDM and RCNM); Bayanga, base camp, tent, night capture 24.X.2008 (ð) (Collector PA) (IDM); Dzanga-Ndoki National Park, Molongo, Sangha river bank, UV trap 14.XI.2010 (3今) (Collector NM and PA) (IDM); Lake 1, base camp, UV trap 26.XI.2010 (3今) (Collector NM and PA) (IDM); Lake 7, UV trap 28.XI.2010 (3今) (Collector NM and PA) (IDM); Lake 1, platform on the canopy 45m, "Azobé", Lophira alata, Ochnaceae, UV trap 29.XI-01.XII.2010 (2ठ) (Collector NM and PA) (IDM); Lake 1, base camp, UV trap 30.XI01-XII.2010 (2今) (Collector NM and PA) (IDM); Lake 1, base camp, laboratory tent, night capture 02.XII.2010 (2今) (Collector NM and PA) (IDM); Dzanga-Sangha Special Reserve, Bayanga, WWF guest house, night capture 21.I.2012 (ठ) (Collector NM and PA) (RCNM); Dzanga-Ndoki National Park, M'Boki, Sangha river bank, UV trap 25.I.2012 (ठ̋) (Collector NM and PA) (IDM); Lake 1, base camp, leaf, day capture 01.II.2012 (ठ̋) (Collector NM and PA) (RCNM); Lake 1, base camp, UV trap 07-10.II.2012 (3今) (Collector NM and PA) (RCNM); Lake 1, base camp, laboratory tent, night capture 11.II.2012 (2 $\left.\delta^{\star}\right)$ (Collector NM and PA) (RCNM); Lake 1, platform on the canopy 38m, "Azobé", Lophira alata, Ochnaceae, UV trap 12.II.2012 (ठ̊) (Collector NM and PA) (RCNM); Lake 1, base camp, UV trap 12.II.2012 (3ðึ) (Collector NM and PA) (RCNM); Lake 1, base camp, laboratory tent, night capture 13-14.II.2012 (2ई) (Collector NM and PA) (RCNM); Lake 3, UV trap 15.II.2012 (ふ) (Collector NM and PA) (RCNM); Lake 1, base camp, laboratory tent, Genitalia Moulin NM0083 $(1 \hat{\jmath})$, barcoding BOLD NMMAN11-410 (1 $\hat{\delta})$, Genitalia Moulin

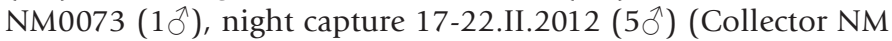
and PA) (IDM and RCNM); Lake 1, platform on the canopy $45 \mathrm{~m}$, "Ayous" Triplochiton scleroxylon, Sterculariaceae, barcoding BOLD NMMAN11-450 (1ठ), UV trap 24.II.2012 (2仓) (Collector NM and PA) (RCNM); Lake 1, base camp, UV trap 25.II.2012 (ㅇ) (Collector NM and PA) (RCNM).

Distribution.-CAR, Congo, Democratic Republic of the Congo, Gabon, Ghana, Guinea, Ivory Coast.

\section{Mantidae, Deromantinae Genus Deromantis Karsch, 1892}

\author{
Deromantis limbalicollis (Karsch, 1892)
}

Karsch 1892a. Ent. Nachr. 18: 7.

Type locality.-Kribi (Cameroon).

Material examined. - CAR, Dzanga-Sangha Special Reserve, Lidjombo, base camp, platform on the canopy 35m, "Azobé" Lophira alata, Ochnaceae, UV trap 02.II.2005 (ð) (Collector PA) (RCNM); Lidjombo, in a little bay, UV trap 06.II.2005 (ठ̊) (Collector PA) (IDM); Dzanga-Ndoki National Park, Lake 1, base camp, Genitalia Moulin NM0080 (1ठ), UV trap 30.I-12.II.2012 (3今) (Collector NM and PA) (IDM and RCNM); Lake 1, base camp, laboratory tent, night capture 24.II.2012 (ठ̊) (Collector NM and PA) (IDM).

Distribution.-Cameroon, CAR, Congo, Gabon.

\section{Mantidae, Omomantinae Genus Omomantis Saussure, 1899}

Omomantis sigma Rehn, 1949

Rehn 1949. Trans. Amer. Ent. Soc. 74: 200.

Type locality._Lukolela (Democratic Republic of the Congo).

Material examined.-CAR, Dzanga-Sangha Special Reserve, between Bayanga and Lidjombo, PK21, UV trap, 16.VI.1998 (3ð) (Collector PA) (IDM); Lidjombo, UV trap 06.II.2005 (ठ) (Collector PA) (RCNM); Bayanga, platform on the canopy $44 \mathrm{~m}$, "Kungu" Piptadenastrium africanum, Fabaceae, UV trap 22-23.X.2008 (4خ) (Collector PA) (IDM); Bayanga, Sangha river bank, UV trap 25.X.2008 (20⿱) (Collector PA) (IDM and RCNM); Dzanga-Ndoki National Park, Lake 1, platform on the canopy 45m, "Azobé" Lophira alata, Ochnaceae, UV trap 01.XII.2010 (ठ̊) (Collector NM and PA) (IDM); Buffalos bay, UV trap 26.I.2012 (2今) (Collector NM and PA) (RCNM); Lake 1, base camp, UV trap 27.I.2012 (ठ) (Collector NM and PA) (RCNM); Lake 1, platform on the canopy 38m, "Azobé" Lophira alata, Ochnaceae, UV trap 10-12.II.2012 (5ठ⿱) (Collector NM and PA) (RCNM); Lake 1, platform on the canopy 45m, "Ayous" Triplochiton scleroxylon, Sterculariaceae, barcoding BOLD NMMAN11-420 and -421, UV trap 15.II.2012 (3今) (Collector NM and PA) (IDM and RCNM); Lake 3, UV trap 15.II.2012 (20) (Collector NM and PA) (RCNM); Lake 1, platform on the canopy 45m, "Ayous" Triplochiton scelroxylon, Sterculariaceae, Genitalia Moulin NM0053 (1ठ), barcoding BOLD NMMAN11-435 (1ठ), UV trap 22.II.2012 (4ठ) (Collector NM and PA) (RCNM); Lake 3, barcoding BOLD NMMAN11-443, UV trap 22.II.2012 (2ठ) (Collector NM and PA) (RCNM); Lake 1, platform on the canopy 45m, "Ayous" Triplochiton scleroxylon, sterculariaceae, barcoding BOLD NMMAN11-455 (1ठ̋), UV trap 23-24.II.2012 (3ß) (Collector NM and PA) (RCNM).

Distribution._CAR, Democratic Republic of the Congo, Gabon. 


\section{Mantidae, Tenoderinae, Tenoderini} Genus Plistospilota Giglio-Tos, 1911

\section{Plistospilota sp1 cf. maxima}

Type locality.--Research required and it is the subject of an upcoming revision.

Material examined.-CAR, Dzanga-Sangha Special Reserve, Bayanga, base camp, barcoding BOLD NMMAN11-427 (1ठ), UV trap 11-25.X.2008 (2ð) (Collector PA) (RCNM); Bayanga, base camp, barcoding BOLD NMMAN11-426 (1亏), leaf, night capture 1824.X.2008 (2今) (Collector PA) (RCNM).

Distribution._Cameroon, CAR, Congo, Ivory Coast ?, Gabon.

\section{Plistospilota sp2 cf. validissima}

Type locality.-Research required and it is the subject of an upcoming revision.

Material examined._CAR, Dzanga-Sangha Special Reserve, Bayanga, UV trap 15-20.II.1986 (ð) (Collector PA) (IDM); between Bayanga and Lidjombo, PK21, UV trap 16.VI.1998 (2今) (Collector PA) (IDM); Lidjombo, in a little bay, UV trap 04.II.2005 (ð) (Collector PA) (IDM); Bayanga, base camp, UV trap 16.X.2008 (2خ̂) (Collector PA) (IDM); Bayanga, base camp, night capture 21.X.2008 (ふ) (Collector PA) (IDM); Bayanga, Sangha river bank, UV trap 25.X.2008 (ð) (Collector PA) (IDM); Dzanga-Ndoki National Park, Molongo, trunk, day capture 15.XI.2010 (ð) (Collector NM and PA) (IDM); Dzanga-Ndoki National Park, base camp, UV trap 24.XI.2010 (3 $\hat{\text { C }) ~(C o l l e c t o r ~ N M ~ a n d ~ P A) ~(I D M ~ a n d ~ R C N M) ; ~ L a k e ~ 7, ~}$ UV trap 28.XI.2010 (ð) (Collector NM and PA) (IDM); Lake 1, base camp, UV trap 28.XI.2010 (ð) (Collector NM and PA) (RCNM); Lake 5, little clearing in the forest, UV trap 29.XI.2010 (ठ̊) (Collector NM and PA) (IDM); Lake 1, platform on the canopy 45m, "Azobé" Lophira alata, Ochnaceae, UV trap 29.XI.2010 (ठ̂) (Collector NM and PA) (IDM); Lake 1, base camp, UV trap 29-30.XI.2010 (4 $\overbrace{}^{\hat{A}}$ (Collector NM and PA) (IDM); Lake 1, platform on the canopy 45m, "Azobé" Lophira alata, Ochnaceae, UV trap 01.XII.2010 (ठ઼) (Collector NM and PA) (RCNM); Dzanga-Sangha Special Reserve, Bayanga, leaf, night capture 21.I.2012 (nymph) (Collector NM and PA) (RCNM); Bayanga, WWF guest house, night capture 21.I.2012 (ठ) (Collector NM and PA) (RCNM); Lake 7, UV trap 03.II.2012 (ठ) (Collector NM and PA) (IDM); Lake 1, base camp, Genitalia Moulin NM0055 (1ð), UV trap 10-11.II.2012 (3ð) (Collector NM and PA) (RCNM); Lake 1, base camp, laboratory tent, night capture 12.II.2012 (nymph) (Collector NM and PA) (RCNM); Lake 1, platform on the canopy 38m, "Azobé" Lophira alata, Ochnaceae, UV trap 12.II.2012 (ふ) (Collector NM and PA) (RCNM); Lake 1, base camp, laboratory tent, barcoding BOLD NMMAN11-419 and -433, night capture 19.II.2012 (20) (Collector NM and PA) (RCNM); Lake 1, base camp, laboratory tent, Genitalia Moulin NM0057, night capture 22.II.2012 (ఓ) (Collector NM and PA) (RCNM); Lake 3, UV trap 22.II.2012 (ð) (Collector NM and PA) (RCNM); Lake 1, base camp, laboratory tent, night capture 27.II.2012 (ð̊) (Collector NM and PA) (RCNM); Lake 7, barcoding BOLD NMMAN11-418, UV trap 01.III.2012 (ふ̋) (Collector NM and PA) (RCNM).

Distribution.-Cameroon, CAR, Democratic Republic of the Congo, Gabon, Ghana, Ivory Coast, Liberia.

\section{Genus Cataspilota Giglio-Tos, 1917}

Note.-This genus is being reviewed.

Cataspilota calabarica Westwood, 1889

Westwood, 1889. Revis. Mantid. p. 35.

Type locality._Calabar (Nigeria).

Material examined.-CAR, Dzanga-Sangha Special Reserve, Bayanga, Sangha river bank, UV trap 09.X.2008 (2今) (Collector PA) (IDM); Bayanga, UV trap 19.X.2008 (ふ̋) (Collector PA) (IDM); Dzanga-Ndoki National Park, Lake 1, platform on the canopy 45m, "Azobé" Lophira alata, Ochnaceae, UV trap 01.XII.2010 (ठ゚) (Collector NM and PA) (RCNM); Lake 1, platform on the canopy $38 \mathrm{~m}$, "Azobé" Lophira alata, Ochnaceae, Genitalia Moulin NM0089, UV trap 03.II.2012 (ठ) (Collector NM and PA) (RCNM); Lake1, base camp, laboratory tent, night capture 13.II.2012 (ð) (Collector NM and PA) (RCNM).

Distribution._Bioko Island, Cameroon, CAR, Equatorial Guinea, Gabon, Liberia, Nigeria.

\section{Cataspilota lolodorfana (Giglio-Tos, 1911)}

Giglio-Tos 1911. Bull. Soc. Ent. Ital. 42: 11.

Type locality._Lolodorf (Cameroon).

Material examined._CAR, Komassa, UV trap 05-07.VIII.1966 (2ふै) (Collector M. Boulard) (MNHN); Salo, Genitalia Roy 1047 (1ठ), UV trap 11.IX.1966 (ô) (Collector M. Boulard) (MNHN); DzangaSangha Special Reserve, Lidjombo, in a little bay, barcoding BOLD NMMAN11-397, UV trap 05.II.2005 (ठ̋) (Collector PA) (RCNM); Bayanga, UV trap 19.X.2008 (ð̋) (Collector PA) (RCNM); Bayanga, platform on the canopy 44m, "Kungu" Piptadenastrium africanum, Fabaceae, UV trap 21-23.X.2008 (20⿱) (Collector PA) (RCNM); Dzanga-Ndoki National Park, Lake 7, UV trap 28.XI.2010 (ð) (Collector NM and PA) (RCNM); Lake 1, base camp, laboratory tent, night capture 02.XII.2010 (2犬) (Collector NM and PA) (RCNM); Lake 1, base Camp, Genitalia Moulin NM0096, UV trap 28.I.2012 ( ఓ) (Collector NM and PA) (RCNM); Lake 1, base Camp, Remote Canopy Trap, UV trap 28.I.2012 (ð)) (Collector NM and PA) (RCNM); Lake 1, base camp, UV trap 02-07.II.2012 $\left(3 \delta^{\hat{*}}\right)$ (Collector NM and PA) (RCNM); Lake 1, platform on the canopy 38m, "Azobé" Lophira alata, Ochnaceae, UV trap 10.II.2012 ( $)$ (Collector NM and PA) (RCNM); Lake 1, base camp, Genitalia Moulin NM0051, UV trap 10.II.2012 (5今) (Collector NM and PA) (RCNM); Lake 1, base camp, laboratory tent, Genitalia Moulin NM0168, night capture 11.II.2012 (ठ઼) (Collector NM and PA) (RCNM); Lake 1, base camp, UV trap 12.II.2012 (11 đ) (Collector NM and PA) (RCNM): Lake 1, UV trap 13.II.2012 (ठ) (Collector NM and PA) (RCNM); Lake 1, base camp, laboratory tent, Genitalia Moulin NM0070 (1ठ), night capture 13-14.II.2012 (7ठै) (Collector NM and PA) (RCNM); Lake 1, platform on the canopy 45m, "Ayous" Triplochiton scleroxylon, Sterculariaceae, UV trap 15.II.2012 (ठ) (Collector NM and PA) (RCNM); Lake 3, Genitalia Moulin NM0054 (1ठ) and NM0056 (1ठ), UV trap 15-16.II.2012 (4ठ) (Collector NM and PA) (RCNM); Lake 1, base camp, day capture 18.II.2012 (ठ̊) (Collector NM and PA) (RCNM); Lake 1, base 
camp, laboratory tent, Genitalia Moulin NM0048 (1ठ), NM0058

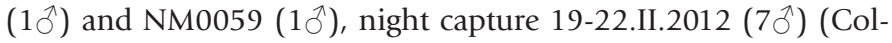
lector NM and PA) (RCNM); Lake 3, Genitalia Moulin NM0167, UV trap 22.II.2012 (ふ) (Collector NM and PA) (RCNM); Lake 1, platform on the canopy 45m, "Ayous" Triplochiton scleroxylon, Sterculariaceae, Genitalia Moulin NM0076, UV trap 24.II.2012 (ふ) (Collector NM and PA) (RCNM).

Distribution.-Cameroon, CAR, Congo, Democratic Republic of the Congo, Gabon, Nigeria.

\section{Cataspilota sp.}

Note.-See discussion about this case in the section on DNA barcoding results.

Type locality.- Research required and it is the subject of an upcoming revision.

Material examined._CAR, Salo, Genitalia Roy 1047, UV trap 11.IX.1966 (ふ) (Collector M. Boulard) (MNHN); Dzanga-Sangha Special Reserve, Lidjombo, in a little bay, barcoding BOLD NMMAN11-396 (1ठ̃), UV trap 05-06.II.2005 (20̂) (Collector PA) (RCNM); Bayanga, platform on the canopy 54m, "Ayous" Triplochiton scleroxylon, Sterculariaceae, UV trap 17-18.X.2008 (2仓) (Collector PA) (RCNM); Bayanga, camp near a "Kungu" Piptadenastrium africanum, Fabaceae, barcoding BOLD NMMAN11-395 (1ठ), UV trap 22.X.2008 (ð) (Collector PA) (RCNM); Bayanga, platform on the canopy $44 \mathrm{~m}$, "Kungu" Piptadenastrium africanum, Fabaceae, Genitalia Moulin NM0090 (1ठ), barcoding BOLD NMMAN11-394 (1ठ) and -406 (1ठ), UV trap 22.X.2008 (2仓) (Collector PA) (RCNM); Dzanga-Ndoki National Park, Lake 1, platform on the canopy 45m, "Azobé" Lophira alata, Ochnaceae, UV trap 01.XII.2010 (2今) (Collector NM and PA) (RCNM); Molongo, Sangha river bank, Genitalia Moulin NM0091, UV trap 24.I.2012 (ð) (Collector NM and PA) (RCNM); Buffalos bay, Genitalia Moulin NM0169, UV trap 26.I.2012 (ठ)) (Collector NM and PA) (RCNM); Lake 1, base camp, laboratory tent, Genitalia Moulin NM0094, night capture 01.II.2012 (ठ) (Collector NM and PA) (RCNM); Lake 7, Genitalia Moulin NM0088, UV trap 03.II.2012 (§) (Collector NM and PA) (RCNM); Lake 1, platform on the canopy 38m, "Azobé" Lophira alata, Ochnaceae, Genitalia Moulin NM0050 (1ठ) and NM0098 (1ठ), barcoding BOLD NMMAN11-408 (1ठ), UV trap 09-10.II.2012 (3ð) (Collector NM and PA) (RCNM); Lake 3, UV trap 16-18.II.2012 (4ठ) (Collector NM and PA) (RCNM); Lake 1, platform on the canopy $45 \mathrm{~m}$, "Ayous" Triplochiton scleroxylon, Sterculariaceae, Genitalia Moulin NM0060

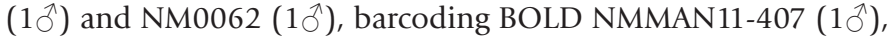
UV trap 22-24.II.2012 (3今) (Collector NM and PA) (RCNM); Lake 3, platform on the canopy 40m, "Sapelli", Entandrophragma cylindricum, Meliaceae, Genitalia Moulin NM0078 (1ठ), UV trap 26.II.2012 ( $\overbrace{}^{\lambda})$ (Collector NM and PA) (RCNM).

\section{Cataspilota tristis (Giglio-Tos, 1911)}

Giglio-Tos 1911. Bull. Soc. Ent. Ital. 42: 6.

Type locality.-Ngoko (Cameroon).

Material examined.—CAR, Komassa, UV trap 07.VIII.1966 (ふ) (Collector M. Boulard) (MNHN); Dzanga-Sangha Special Reserve, between Bayanga and Lidjombo, PK15, UV trap 31.V.1998 (ふ) (Collector PA) (RCNM); Bayanga, base camp, UV trap 17.X.2008 (ठ) (Collector PA) (RCNM); Bayanga, camp 2, leaf, night capture 24.X.2008 (ठ̊) (Collector PA) (IDM); Dzanga-Ndoki National Park, Lake 1, base camp, UV trap 26.XI.2010 (ふ) (Collector NM and PA) (RCNM); Lake 5, little clearing in the forest, UV trap 29.XI.2010 (2へ) (Collector NM and PA) (IDM); Lake 1, base camp, Genitalia Moulin NM0095, Remote Canopy Trap, UV trap 28.I.2012 (ठ) (Collector NM and PA) (RCNM); Lake 1, base camp, laboratory tent, night capture 13.II.2012 (ठ) (Collector NM and PA) (RCNM).

Distribution. -Cameroon, CAR, Gabon.

\section{Genus Polyspilota Burmeister, 1838}

Polyspilota aeruginosa (Goeze, 1778)

Goeze 1778. Ent. Beitrag. 2: 35.

Type locality.-Unknown.

Material examined.-CAR, Dzanga-Sangha Reserve Special, between Bayanga and Lidjombo, PK15, UV trap 31.V.1998 (우) (Collector PA) (RCNM); between Bayanga and Lidjombo, PK21, UV trap 15.VI.1998 (ठ) (Collector PA) (IDM); Dzanga-Ndoki National Park, Molongo, UV trap 15.XI.2010 (2خ) (Collector NM and PA) (IDM); Lake 1, platform on the canopy 45m, "Azobé" Lophira

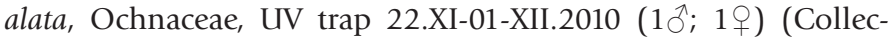
tor NM and PA) (RCNM); Bambio, leaf, day capture 24.I.2012 (4 nymph) (Collector NM and PA) (RCNM); Dzanga-Ndoki National Park, Lake 6, leaf, day capture 03.II.2012 (nymph) (Collector NM and PA) (RCNM); Dzanga-Sangha Special Reserve, Bayanga, Doli Lodge, leaf, barcoding BOLD NMMAN11-401, night capture 16.II.2012 (q) (Collector NM and PA); Dzanga-Ndoki National Park, Lake 1, base camp, laboratory tent, barcoding BOLD NMMAN11-436, night capture 20.II.2012 (§) (Collector NM and PA) (RCNM); Lake 3, barcoding BOLD NMMAN11-447, UV trap 22.II.2012 ( + ) (Collector NM and PA) (RCNM); Lake 1, platform on the canopy 45m, "Ayous" Triplochiton scleroxylon, Sterculariaceae, barcoding BOLD NMMAN11-444 (1ð) and -501 (1ठ), UV trap 15-24.II.2012 (2今) (Collector NM and PA) (RCNM); Molongo, Sangha river bank, leaf, day capture 05.III.2012 (2 nymph) (Collector NM and PA) (RCNM); Molongo, Sangha river bank, leaf, barcoding BOLD NMMAN11-442, night capture 08.III.2012 (す) (Collector NM and PA) (RCNM).

Distribution.-Angola, Cameroon, Cape Verde, CAR, Comoros, Congo, Democratic Republic of the Congo, Ethiopia, Gabon, Ghana, Guinea, Kenya, Liberia, Madagascar, Namibia, South Africa, Tanzania, Uganda, Zanzibar, Zimbabwe.

\section{Polyspilota pavani La Greca, 1966}

La Greca 1966. Frag. ent. 4: 100.

Type locality.-La Maboke (CAR).

Material examined.-CAR, Dzanga-Sangha Special Reserve, Bayanga, platform on the canopy 54m, "Ayous" Triplochiton scleroxylon, Sterculariaceae, UV trap 13-17.X.2008 (2ठ) (Collector PA) 
(IDM); Dzanga-Ndoki National Park, Lake 1, base camp, UV trap 29.XI.2010 (ठ̋) (Collector NM and PA) (IDM); Dzanga-Sangha Special Reserve, Bayanga, WWF guest house, night capture 21.I.2012 (ð) (Collector NM and PA) (RCNM); Dzanga-Ndoki National Park, Lake 1, platform on the canopy 38m, "Azobé" Lophira alata, Ochnaceae, Genitalia Moulin NM0097, UV trap 10.II.2012 (ð) (Collector NM and PA) (RCNM).

Distribution._CAR, Gabon.

\section{Genus Prohierodula Bolivar, 1908}

Prohierodula grassei Roy, 1965

Roy 1965. Biologia Gabon. 1: 289.

Type locality.-Belinga (Gabon).

Material examined._CAR, Dzanga-Sangha Special Reserve, Lidjombo, in a little bay, UV trap 04.II.2005 (ठ) (Collector PA) (IDM); Bayanga, platform on the canopy 44m, "Kungu" Piptadenastrium africanum, Fabaceae, UV trap 21.X.2008 (2ઈ) (Collector PA) (IDM); Bayanga, base camp, tent, night capture 21.X.2008 (ð) (Collector PA) (IDM); Dzanga-Ndoki National Park, Lake 5, in a little clearing, UV trap 29.XI.2010 (ठ) (Collector NM and PA) (RCNM); Lake 1, base camp, UV trap 09-12.II.2012 (6 ${ }^{\AA}$ ) (Collector NM and PA) (RCNM); Lake 1, base camp, laboratory tent, barcoding BOLD NMMAN11-432 (1ふ) and -449 (1ふ), night capture 13-24.II.2012 (4仓) (Collector NM and PA) (RCNM); Lake 1, platform on the canopy 45m, "Ayous" Triplochiton scleroxylon, Sterculariaceae, UV trap 24.II.2012 (ठ) (Collector NM and PA) (RCNM).

Distribution._CAR, Democratic Republic of the Congo, Gabon.

\section{Prohierodula laticollis (Karsch, 1892)}

Karsch 1892b. Ent. Nachr. 18: 147.

Type locality._Barombi (Cameroon).

Material examined._CAR, Komassa, UV trap 05-13.VIII.1966 (9今); Dzanga-Sangha Special Reserve, between Bayanga and Lidjombo, PK21, UV trap 15.VI.1998 (ठ઼) (Collector PA) (IDM); Between Bayanga and Lidjombo, PK21, UV trap 16.VI.1998 (2ङ) (Collector PA) (IDM); Lidjombo, in a little bay, UV trap 06.II.2005 (ふ) (Collector PA) (IDM); Bayanga, transit camp near Songo waterfalls, UV trap 10.X.2008 (ふ) (Collector PA) (IDM); Bayanga, platform on the canopy 54m, "Ayous" Triplochiton scleroxylon, Sterculariaceae, UV trap 17.X.2008 (3ð) (Collector PA) (IDM); Bayanga, base camp, UV trap 18.X.2008 (ð̋) (Collector PA) (IDM); Bayanga, base camp, tent, night capture 20.X.2008 (ð) (Collector PA) (IDM); Bayanga, base camp, UV trap 21.X.2008 (ð)) (Collector PA) (IDM); Bayanga, Sangha river bank, UV trap 25.X.2008

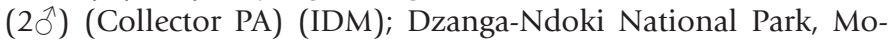
longo, Sangha river bank, UV trap 14.XI.2010 (2 $\hat{O}^{\hat{)}}$ ) (Collector NM and PA) (IDM); Lake 1, base camp, laboratory tent, night capture 20.XI.2010 (§) (Collector NM and PA) (IDM); Lake 1, platform on the canopy 35m, "Azobé" Lophira alata, Ochnaceae, UV trap 23-25.XI.2010 (2へ) (Collector NM and PA) (IDM); Lake 1, base camp, UV trap 26.XI.2010 (2ઈ) (Collector NM and PA) (IDM); Lake 1, base camp, laboratory tent, night capture 26.XI.2010 (2§)
(Collector NM and PA) (IDM); Lake 4, leaf, day capture 27.XI.2010 (ठ) (Collector NM and PA) (IDM); Lake 7, UV trap 28.XI.2010 (6 ) (Collector NM and PA) (IDM); Lake 1, base camp, UV trap 28.XI.2010 (2今) (Collector NM and PA) (IDM); Lake 1, platform on the canopy 35m, "Azobé" Lophira alata, Ochnaceae, UV trap 28.XI.2010 (ð) (Collector NM and PA) (IDM); Lake 1, base camp, laboratory tent, night capture 28.XI.2010 (7 $\hat{)}$ ) (Collector NM and PA) (IDM); Lake 5, in a little clearing, UV trap 29.XI.2010 $(5 \hat{)})$ (Collector NM and PA) (IDM); Lake 1, base camp, UV trap 29.XI.2010 (3今) (Collector NM and PA) (IDM and RCNM); Lake 1, base camp, laboratory tent, night capture 29.XI.2010 (2今) (Collector NM and PA) (IDM and RCNM); Lake 1, platform on the canopy 45m, "Azobé" Lophira alata, Ochnaceae, UV trap 30.XI-01. XII.2010 (5ð) (Collector NM and PA) (IDM); Lake 1, base camp, laboratory tent, night capture 02.XII.2010 (ठ̋) (Collector NM and PA) (IDM); Lake 1, UV trap 02.XII.2010 (ठ) (Collector NM and PA) (IDM); Molongo, Sangha river bank, UV trap 25.I.2012 (2ð) (Collector NM and PA) (RCNM); Buffalos bay, UV trap 26.I.2012 $\left(2 \hat{O}^{\Uparrow}\right)$ (Collector NM and PA) (RCNM); Lake 1, base camp, UV trap 27-30.I.2012 (5 $\hat{\text { ) }}$ (Collector NM and PA) (IDM and RCNM); Lake 7, UV trap 02.II.2012 (2仓) (Collector NM and PA) (IDM); Lake 1, near weir, leaf, day capture 02.II.2012 (ठ) (Collector NM and PA) (RCNM); Lake 7, leaf, night capture 03.II.2012 (ð̊) (Collector NM and PA) (RCNM); Lake 1, platform on the canopy 38m, "Azobé" Lophira alata, Ochnaceae, UV trap 07.II.2012 (ठ̊) (Collector NM and PA) (RCNM); Lake 1, base camp, UV trap 09.II.2012 (3ڤึ) (Collector NM and PA) (RCNM); Lake 1, platform on the canopy 38m, "Azobé" Lophira alata, Ochnaceae, UV trap 10.II.2012 (3ð) (Collector NM and PA) (IDM and RCNM); Lake 1, base camp, UV trap 10.II.2012 (7ð) (Collector NM and PA) (RCNM); Lake 1, base camp, laboratory tent, night capture 11.II.2012 (6 $\delta^{\Uparrow}$ ) (Collector NM and PA) (RCNM); Lake 1, platform on the canopy $38 \mathrm{~m}$, "Azobé" Lophira alata, Ochnaceae, UV trap 12.II.2012 (す) (Collector NM and PA) (RCNM); Lake 1, base camp, UV trap 12.II.2012

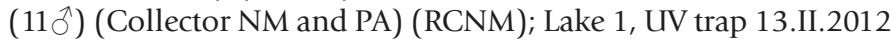
(2仓) (Collector NM and PA) (RCNM); Lake 1, base camp, laboratory tent, night capture 13-14.II.2012 (10§) (Collector NM and

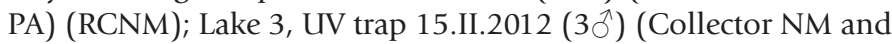
PA) (RCNM); Lake 1, base camp, laboratory tent, night capture 15.II.2012 (ठ) (Collector NM and PA) (RCNM); Lake 3, UV trap

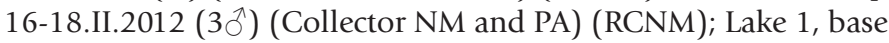
camp, UV trap 19.II.2012 (ð) (Collector NM and PA) (RCNM); Lake 1, base camp, laboratory tent, night capture 19.II.2012 (12خ) (Collector NM and PA) (RCNM); Lake 1, base camp, laboratory tent, night capture 20.II.2012 (5ळ) (Collector NM and PA) (RCNM); Lake 1, leaf, day capture 21.II.2012 (ठ) (Collector NM and PA) (RCNM); Lake 1, base camp, UV trap 21.II.2012 (ठ̋) (Collector NM and PA) (RCNM); Lake 1, platform on the canopy 45m, "Ayous" Triplochiton scleroxylon, Sterculariaceae, barcoding BOLD NMAN11-441 (1ठ), UV trap 22.II.2012 (4仓) (Collector NM and PA) (RCNM); Lake 3, UV trap 22.II.2012 (4) (Collector NM and PA) (RCNM); Lake 1, base camp, UV trap 22.II.2012 (ठ̊) (Collector NM and PA) (RCNM); Lake 1, platform on the canopy $45 \mathrm{~m}$, "Ayous" Triplochiton scleroxylon, Sterculariaceae, UV trap 24.II.2012 (ठ) (Collector NM and PA) (RCNM); Lake 1, base camp, UV trap 26.II.2012 (2§) (Collector NM and PA) (RCNM); Lake 1, base camp, laboratory tent, night capture 27.II.2012 (ð)) (Collector NM and PA) (RCNM); Lake 7, UV trap 01.III.2012 (ठ̋) (Collector NM and PA) (RCNM).

Distribution.-Cameroon, CAR, Democratic Republic of the Congo, Gabon. 


\section{Prohierodula mundamensis Giglio-Tos, 1911}

Giglio-Tos 1911. Bull. Soc. Ent. Ital. 42: 37.

Type locality._Mundame (Cameroon).

Material examined.-CAR, Dzanga-Sangha Special Reserve, Lidjombo, UV trap 14.II.2005 (ð) (Collector PA) (IDM).

Distribution.-Cameroon, CAR, Congo, Gabon.

Prohierodula picta (Gerstaecker, 1883)

Gerstaecker 1883. Mitt. Naturw. Ver. Neu-Vorpomm. 14: 88.

Type locality._-Victoria (Cameroon).

Material examined.-CAR, Dzanga-Ndoki National Park, Lake 1, base camp, UV trap 10.II.2012 (ठ) (Collector NM and PA) (RCNM).

Distribution.-Cameroon, CAR, Equatorial Guinea, Gabon.

\section{Prohierodula viridimarginata La Greca, 1956}

La Greca 1956. Annuar. Inst. Mus. Zool. Univ. Napoli 8(11): 18.

Type locality.-Epulu, Uele Ibembo (Democratic Republic of the Congo).

Material examined.-CAR, Komassa, UV trap 07.VIII.1966 (2ð) (Collector M. Boulard) (MNHN); Dzanga-Sangha Special Reserve, Lidjombo, base camp, Sangha river bank, day capture 30.I.2005 (つ) (Collector PA) (IDM); Bayanga, base camp, UV trap 13-20.X.2008 (4仓̃) (Collector PA) (IDM); Dzanga-Ndoki National Park, Lake 1, UV trap 19.XI.2010 (ð) (Collector NM and PA) (RCNM); Lake 1, base camp, UV trap 24.XI.2010 (2ð) (Collector NM and PA) (IDM and RCNM); Lake 7, UV trap 27.XI.2010 (ठ̊) (Collector NM and PA) (IDM); Lake 1, base camp, laboratory tent, night capture 28-29.XI.2010 (3今) (Collector NM and PA) (IDM and RCNM); M'Boki, Sangha river bank, UV trap 24.I.2012 (ð)) (Collector NM and PA) (RCNM); Lake 1, base camp, laboratory tent, night capture 01.II.2012 ( $)$ (Collector NM and PA) (RCNM); Lake 1, Base camp, UV trap 02.II.2012 (3ठ) (Collector NM and PA) (RCNM); Lake 7, UV trap 03.II.2012 (ð) (Collector NM and PA) (RCNM); Lake 1, base camp, UV trap 03-12.II.2012 (5今) (Collector NM and PA) (IDM and RCNM); Lake 1, base camp, laboratory tent, barcoding BOLD-NMMAN11-422 (1ठ) and -502 (1仓), night capture 13-14.II.2012 (4خ) (Collector NM and PA) (RCNM); Lake 3, leaf, day capture 24.II.2012 (ㅇ) (Collector NM and PA) (RCNM).

Distribution._CAR, Democratic Republic of the Congo, Gabon.

\section{Mantidae, Tenoderinae, Paramantini Genus Alalomantis Giglio-Tos, 1917}

Alalomantis muta (Wood-Mason, 1882)

Wood-Mason 1882. J. Asiat. Soc. Bengal 51: 30.

Type locality._Buea (Cameroon).
Material examined.-CAR, Dzanga-Sangha Special Reserve, Lidjombo, in a little bay, UV trap 06.II.2005 (ठ) (Collector PA) (RCNM); Dzanga-Ndoki National Park, Molongo, leaf, day capture 15.XI.2010 (ㅇ) (Collector NM and PA) (IDM); Molongo, UV trap 15.XI.2010 (ठ઼) (Collector NM and PA) (RCNM); Molongo, leaf, night capture 04.XII.2010 (q) (Collector NM and PA) (RCNM); Molongo, Sangha river bank, UV trap 24.I.2012 (ふ) (Collector NM and PA) (RCNM).

Distribution._Angola, Bioko Island, Cameroon, CAR, Democratic Republic of the Congo, Gabon, Uganda.

\section{Genus Sphodromantis Stal, 1871}

Most recent genus revision (Roy 2010).

\section{Sphodromantis balachowskyi La Greca, 1967}

La Greca 1967. Ann. Ist. Mus. Zool. Univ. Napoli 18(1): 24.

Type locality. - Yangambi-Stanleyville=Kisangani (Democratic Republic of the Congo).

Material examined.-CAR, Dzanga-Ndoki National Park, Lake 1, platform on the canopy 45m, "Azobé" Lophira alata, Ochnaceae, UV trap 24.XI.2010 (ठ̂) (Collector NM and PA) (RCNM); Lake 1, weir, leaf, day capture 26.XI.2010 (ð) (Collector NM and PA) (IDM); Lake 1, platform on the canopy 45m, "Azobé" Lophira alata, Ochnaceae, UV trap 28.XI.2010 (ठ) (Collector NM and PA) (IDM); Lake 5, little clearing in the forest, UV trap 29.XI.2010 (ठ) (Collector NM and PA) (RCNM); Lake 1, platform on the canopy 45m, "Azobé" Lophira alata, Ochnaceae, UV trap 30.XI-01.XII.2010 (3今) (Collector NM and PA) (IDM and RCNM); Lake 1, platform on the canopy 38m, "Azobé" Lophira alata, Ochnaceae, UV trap 07-10.II.2012 (2今) (Collector NM and PA) (RCNM); Lake 1, platform on the canopy 45m, "Ayous" Triplochiton scleroxylon, Sterculariaceae, barcoding BOLD-NMMAN11-438 and -451, UV trap 2223.II.2012 (2仓) (Collector NM and PA) (RCNM).

Distribution.-CAR, Democratic Republic of the Congo, Gabon.

Sphodromantis gracilicollis centroccidentalis Roy, 2010

Roy 2010. Bull. Soc. Entomo. France, 115(3): 345-366.

Type locality._La Maboke (CAR).

Material examined.-CAR, Dzanga-Sangha Special Reserve, Bayanga, UV trap 25.XII.1984 (ठ઼) (Collector PA) (IDM); Dzanga-Ndoki National Park, Lake 1, platform on the canopy 45m, "Azobé" Lophira alata, Ochnaceae, UV trap 29.XI.2010 (ठ゚) (Collector NM and PA) (IDM); Lake 1, base camp, laboratory tent, night capture 02.XII.2010 (ठ) (Collector NM and PA) (RCNM); Dzanga-Sangha Special Reserve, Bayanga, WWF guest house, night capture 21.I.2012 (20) (Collector NM and PA) (IDM and RCNM); Dzanga-Ndoki National Park, Buffalos bay, UV trap 26.I.2012 (ふ઼) (Collector NM and PA) (RCNM); Lake 1, base camp, trunk, night capture 27.I.2012 (ふ) (Collector NM and PA) (RCNM); Lake 1, platform on the canopy 38m, "Azobé" Lophira alata, Ochnaceae, barcoding BOLD LopeMAN14-214 (1ठ), UV trap 04-10.II.2012 (3今) (Collector NM and PA) (RCNM); Lake 1, base camp, labo- 
ratory tent, night capture 11.II.2012 (ð) (Collector NM and PA) (RCNM); Lake 1, platform on the canopy 38m, "Azobé" Lophira alata, Ochnaceae, UV trap 12.II.2012 (ð) (Collector NM and PA) (RCNM); Lake 1, base camp, Genitalia Moulin NM0047, UV trap 12.II.2012 (ठ) (Collector NM and PA) (RCNM); Lake 3, barcoding BOLD-NMMAN11-448, UV trap 15.II.2012 (ठ̋) (Collector NM and PA) (RCNM); Dzanga-Sangha Special Reserve, Bayanga, Doli Lodge, barcoding BOLD-NMMAN11-403, night capture 16.II.2012 (ठ) (Collector J. P. Maurel) (RCNM); Dzanga-Ndoki National Park, Lake 1, base camp, laboratory tent, Genitalia Moulin NM0049, night capture 19.II.2012 (ठ) (Collector NM and PA) (RCNM).

Distribution._Cameroon, CAR, Gabon.

\section{Sphodromantis lineola pinguis La Greca, 1967}

La Greca 1967. Ann. Ist. Mus. Zool. Univ. Napoli 18: 14

Type locality._Brazzaville, Stanleyville, Mbila, Yangambi (Congo). More research required (Roy, com. pers.).

Material examined.-CAR, Dzanga-Sangha Special Reserve, Bayanga, UV trap 1984 (ð) (Collector PA) (IDM); Bayanga, Sangha river bank, UV trap 09.X.2008 (ð) (Collector PA) (IDM); Bayanga, WWF guest house, night capture 21.I.2012 (2^) (Collector NM and PA) (IDM and RCNM); Bambio, leaf, day capture 24.I.2012 (nymph) (Collector NM and PA) (RCNM).

Distribution.-Angola, CAR, Cameroon, Congo, Democratic Republic of the Congo, Gabon.

\section{Mantidae, Hierodulinae Genus Tismomorpha Roy, 1973}

Tismomorpha vitripennis (Bolivar, 1908)

Bolivar 1908a. Mems R. Soc. Esp. Hist. nat. 1: 471.

Type locality.-Eosung, Bakossi Mountains Wildlife Reserve (Cameroon)

Material examined.-CAR, Komassa, UV trap 05-07.VIII.1966 (2ð) (Collector M. Boulard) (MNHN); Dzanga-Sangha Special Reserve, Lidjombo, in a little bay, UV trap 05-07.II.2005 (2仓) (Collector PA) (IDM); Bayanga, base camp, UV trap 16.X.2008 (20̂) (Collector PA) (IDM); Bayanga, base camp, tent, night capture 18-24.X.2008 $(3 \hat{\jmath})$ (Collector PA) (IDM); Dzanga-Ndoki National Park, Lake 1, base camp, UV trap 26.XI.2010 (2ठ) (Collector NM and PA) (IDM and RCNM); Lake 1, base camp, laboratory tent, night capture 2830.XI.2010 (2§) (Collector NM and PA) (IDM and RCNM); Lake 1, base camp, UV trap 02.XII.2010 (ठో) (Collector NM and PA) (IDM); Lake 1, base camp, Remote Canopy Trap, UV trap 28.I.2012 (ふ) (Collector NM and PA) (RCNM); Lake 1, base camp, UV trap 12.II.2012 (2犬) (Collector NM and PA) (RCNM); Lake 1, base camp, laboratory tent, barcoding BOLD NMMAN11-417 (1ठ), night capture 13-19.II.2012 (5 ) (Collector NM and PA) (RCNM); Lake 1, base camp, barcoding BOLD NMMAN11-431 (1 $\hat{\jmath})$, UV trap 26.II.2012 (ð) (Collector NM and PA) (RCNM).

Distribution.-Cameroon, CAR, Gabon.

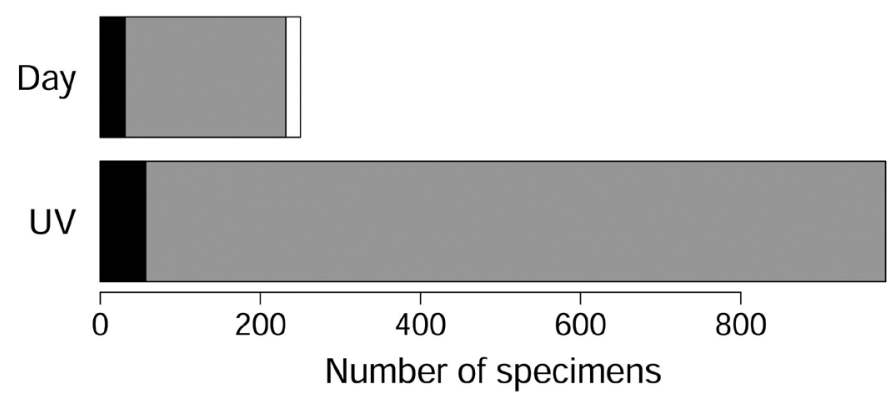

Fig. 5. Number of mantid specimens collected with the two main sampling methods: day capture (Day) and trapping with UV lights at night (UV); black chart $=$ females; grey chart = males; white chart $=$ nymphs.

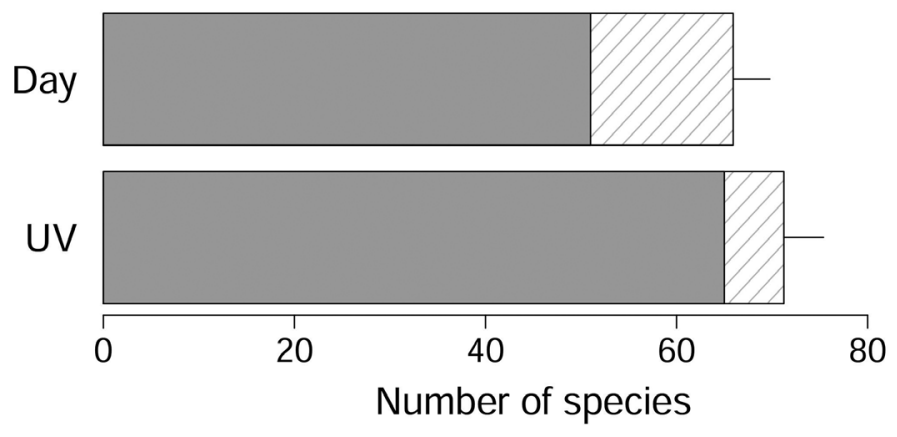

Fig. 6. Specific richness of Mantodea collected with two main sampling methods; day capture (Day) and trapping with UV light at night (UV); grey chart = observed richness; dashed chart = estimated richness (ACE index) and associated standard deviation.

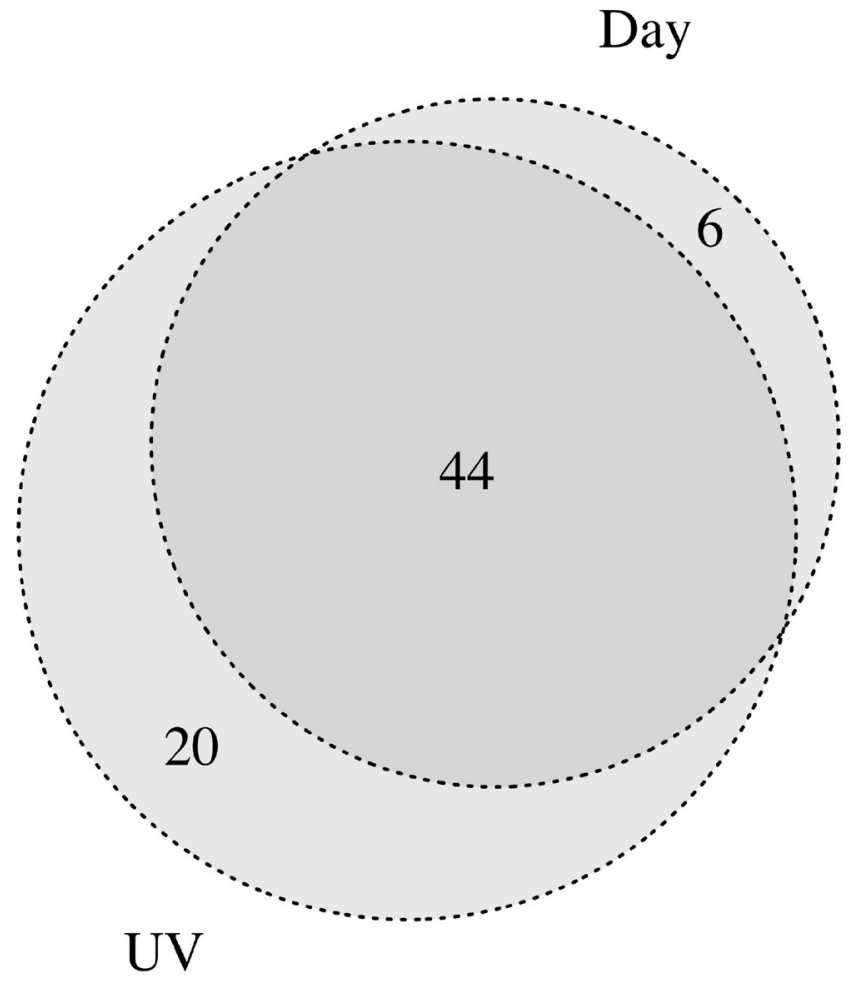

Fig. 7. Number of mantid species collected with the two main sampling methods: day capture (Day) and light trapping (UV). 


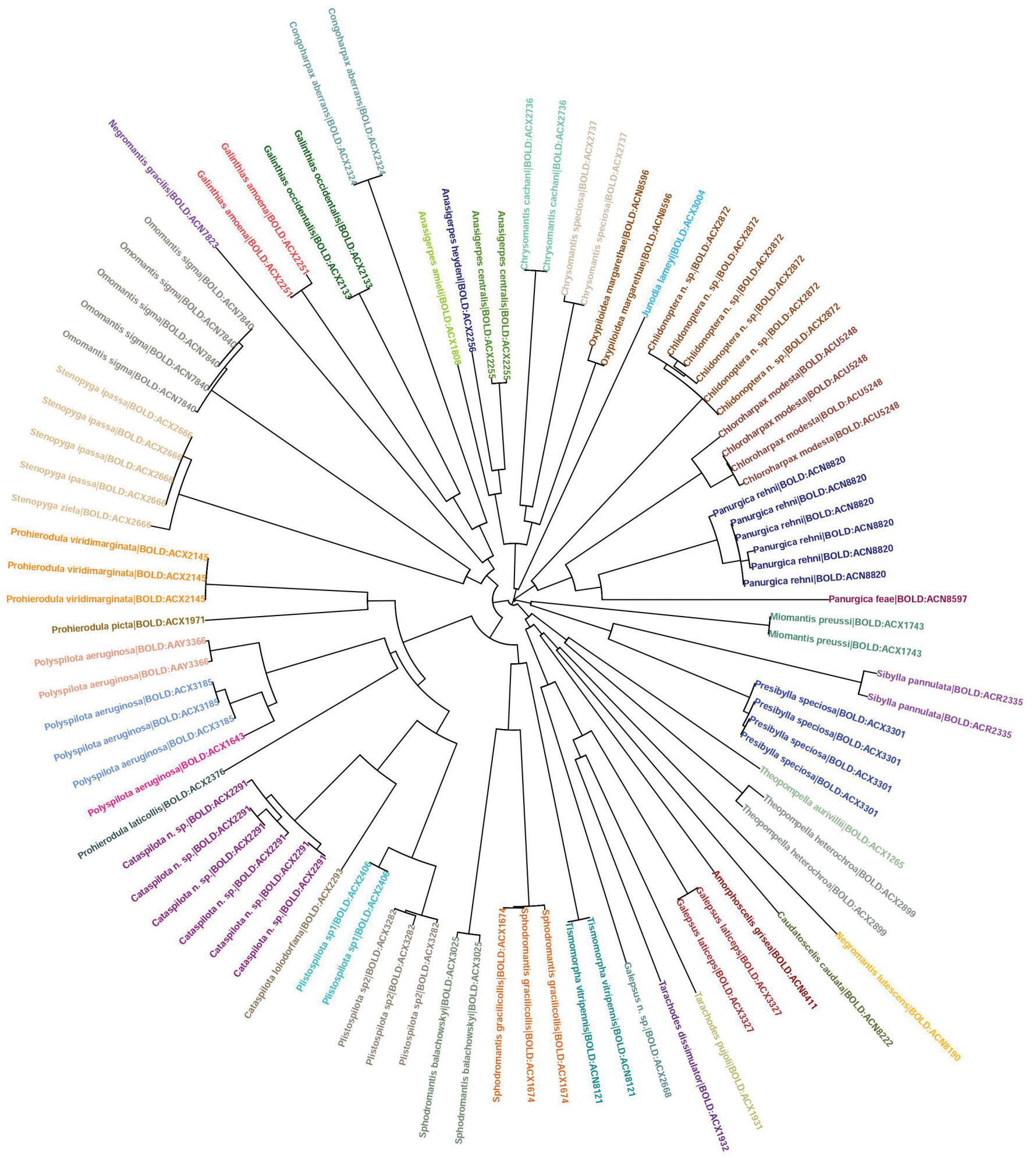

Fig. 8. Neighbor-joining tree of DNA barcodes (COI) obtained from BOLD 4.0. beta using the Kimura 2 parameter for distance model. Filters: sequence length $>200 \mathrm{bp}$. 


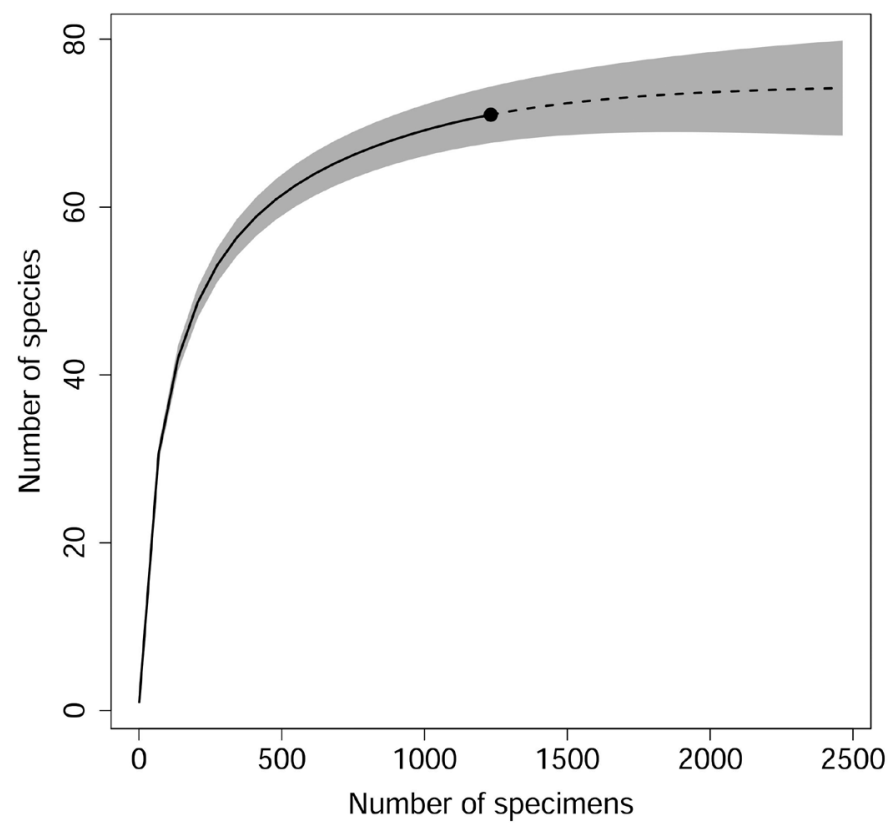

Fig. 9. Rarefaction curve of Mantodea from Sangha-Mbaere Region (black curve) with an extrapolation in a hypothetical situation where sampling effort would be doubled (dashed curve); grey area $=$ confidence interval

Sampling method efficiency.-Light trapping was the most efficient sampling method, with almost 1000 specimens captured, while day collecting only resulted in the collection of 250 specimens (Fig. 5). As for orthopterans, we observed that many mantises were attracted more efficiently by diffuse light (camp lamp in tents, classic neon, low energy consumption lamps) than by the 250W UV light traditionally used in light traps for moths and beetles. As expected, the proportion of females and nymphs was higher during the day than with light traps, and interestingly the number of species collected with both methods was not significantly different (Fig. 6). Sampling completeness, represented by the $\%$ of estimated richness that has been observed in the samples, was higher for light trapping (ca. $92 \%$ ) than for day collecting (ca. 74\%), suggesting that additional sampling effort with this last method would be necessary to reach an accurate estimation of the mantid community diversity. Figure 7 shows the number of species caught specifically for each method.

DNA barcoding versus traditional taxonomy.-A total of 94 sequences of more than $200^{\circ} \mathrm{bp}$ were obtained from 119 specimens analysed. Except in a few cases, the delimitation of taxonomic units on the basis of genetic information contained in the barcodes was congruent with the species limits acknowledged by traditional taxonomy (Fig. 8).

Diversity patterns of praying mantids. - The rarefaction curve obtained for the whole data set (Fig. 9) indicates that the sampling effort was sufficient to provide a representative picture of Mantodea diversity at a regional scale. As exemplified by this figure, doubling the number of collected specimens would only result in the addition to the checklist of a small number of new species. Figure 10 shows diversity patterns in the three major geographical units of the study region, i.e. Dzanga-Ndoki National Park, Dzanga-Sangha Special Reserve and the rest of SanghaMbaere Region.

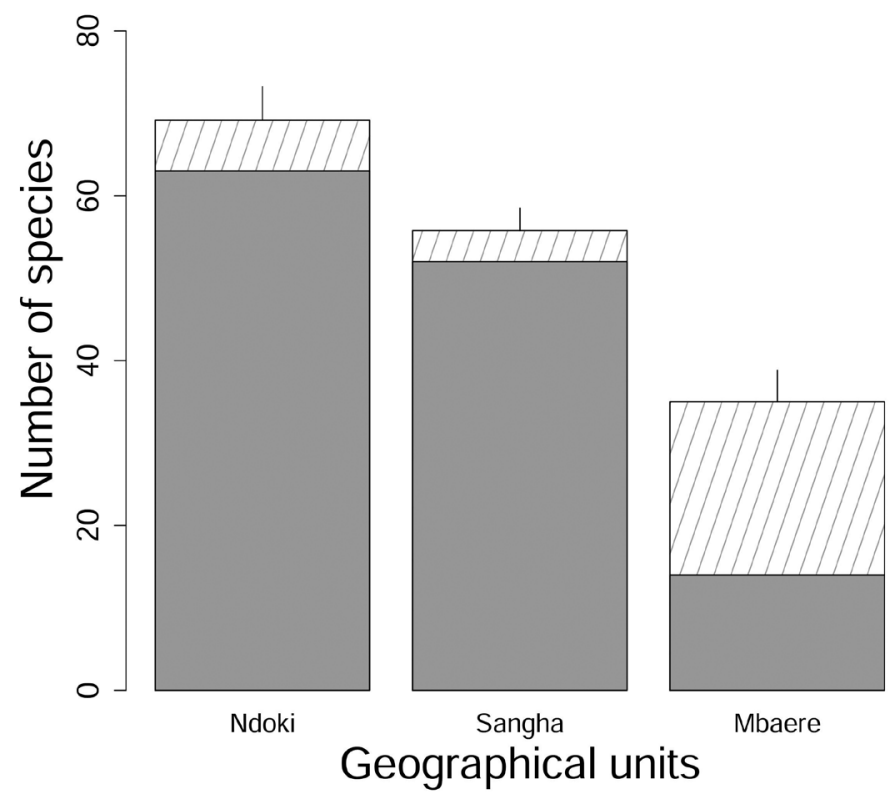

Fig. 10. Species richness in the three zones studied: Ndoki = Dzanga-Ndoki National Park zone; Sangha = Dzanga-Sangha Special Reserve zone; Mbaere $=$ Rest of the Sangha-Mbaere Region; grey chart = observed number of species; dashed chart = estimated richness (ACE index) and associated standard deviation.

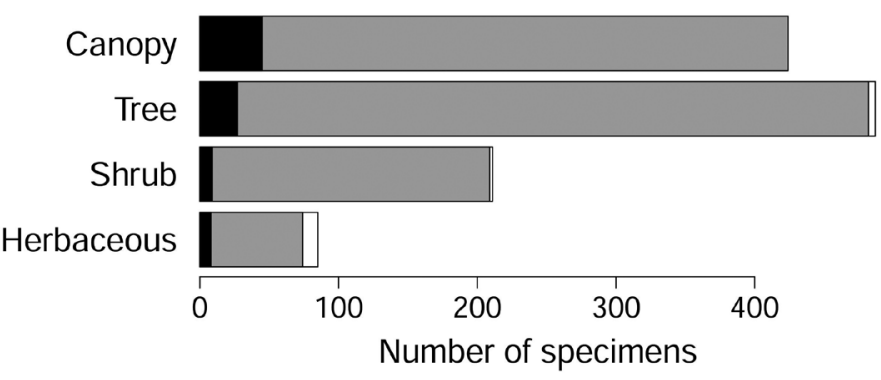

Fig. 11. Number of collected specimens in the different vegetation strata; black = females; grey = males; white $=$ nymphs.

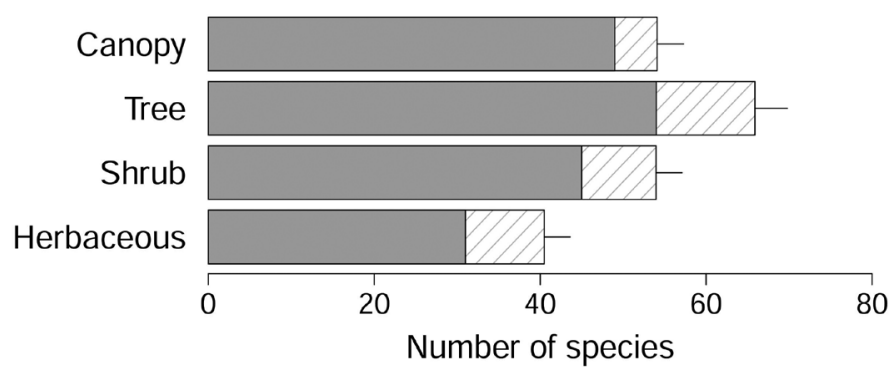

Fig. 12. Specific richness in the different vegetation strata; grey $=$ observed richness; dashed chart = estimated richness (ACE index) and associated standard deviation.

The higher observed and estimated species richness was found in the Dzanga-Ndoki NP, where the number of species was close to the number observed at the regional scale, meaning that most of the regional species pool was present in this area (Fig. 10). 


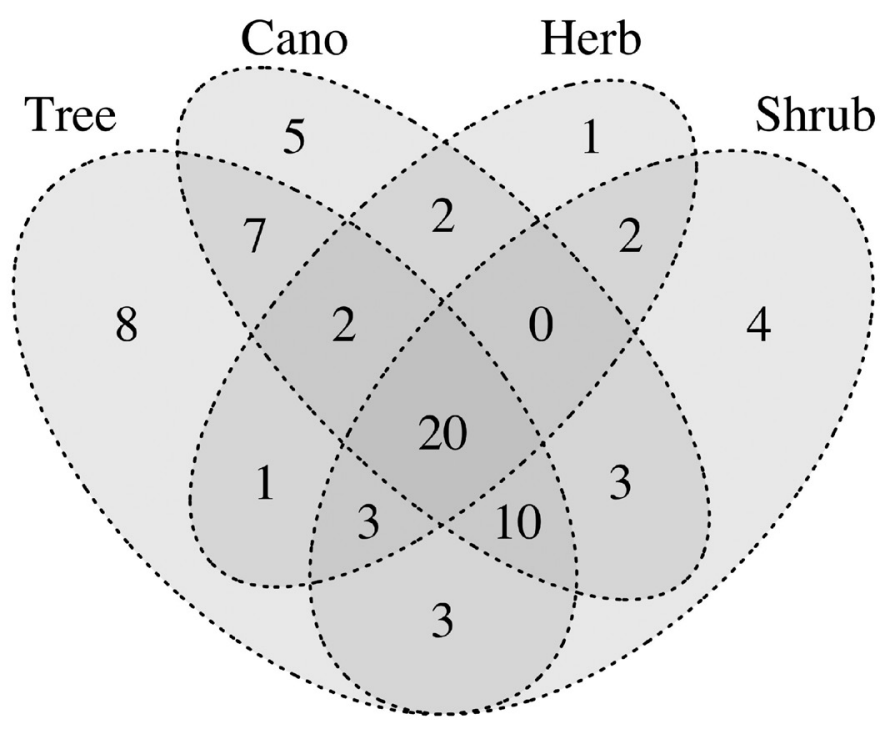

Fig. 13. Distribution of the species in the different vegetation strata.

Vegetation structure.-Figures 11 to 13 present the number of individuals and the diversity of mantids collected along a height gradient from the herbaceous layer to the top canopy. Most of the mantids captured were males because the main method of surveying was through the use of light traps. However, both in top canopy and in the tree stratum, a higher proportion of females were collected by active search and light trap, maybe because UV light was placed nearer to the microhabitats where females resided. The number of specimens found in the soil and herbaceous strata was lower than in other strata because the study area is largely situated in a forest context (Fig. 11). Both observed and estimated species richness were the highest between the shrub layer and the top canopy). However, a significant number of species was found in the herbaceous surroundings and edges of the swamps and lakes (Fig. 12). Each stratum of the vegetation harbors species in a specific way but the species also overlap somewhat in different vegetation types (Fig. 13).

\section{Discussion}

The data obtained during this work provide new insights into our knowledge of the diversity of praying mantids in the SanghaMbaere region and by extension in CAR. At present, mass data from the 1960s exist for La Maboke-Boukoko research station in the Lobaye region but are not yet published. The work presented here also allowed the establishment of a vast reference library of DNA barcodes (COI) for the mantids of Central Africa.

The different sampling methods carried out in the different field surveys provided us with a level of sampling effort that was rarely attained in past studies. It is worth reporting that 6 species were only observed during the day and 20 others only at night, which clearly illustrates the importance of combining different sampling approaches.

The lower richness observed in the Dzanga-Sangha SR could be explained by a difference in forest composition with, for instance, a higher proportion of secondary forests compared with Dzanga-Ndoki NP. Even if a higher sampling effort in DzangaNdoki NP could have resulted in a higher number of observed species, it could hardly explain the difference in the estimated rich- ness between the two regions. The low diversity found in the rest of Sangha-Mbaere Region is certainly linked to a lack of sampling intensity in this area.

In the genus Chlidonoptera, barcoding diagnoses separate species from specimens with very slight differences in habitus and genitalia. Individuals from Sangha-Mbaere are closely related to Chlidonoptera vexillum, which was described from Cameroon and is known from other regions of Central Africa, and Chlidonoptera lestoni which to date is known only from Ghana. A similar case was found within the genus Cataspilota, where DNA barcodes confirmed slight but consistent morphological differences observed between specimens from Sangha-Mbaere and those of Cataspilota lolodorfana from Cameroon. DNA barcodes also support the existence of a new Galepsus sp., which is clearly distinguishable from all other species within this genus on the basis of habitus and genitalia. The case of Plistospilota sp. deserves more attention, as morphological identifications within this genus are often difficult, whereas barcoding seems to clearly separate different BINs. For instance, specimens from Sangha-Mbaere could belong to either Plistospilota maxima or Plistospilota validissima but it was impossible to determine due to the very short and weakly informative original descriptions of both of these closely related species. Two other cases that merit consideration further are represented by Polyspilota aeruginosa, which is subdivided in several BINs while no morphological differences could be found to support these divisions, and by Stenopyga ziela that was grouped with specimens of Stenopyga ipassa in a single BIN, whereas both species are unambiguously differentiated either by their habitus or genitalia.

Although reaching exhaustivity in a biodiversity survey for a group of tropical arthropods remains an unattainable objective, our work produced an extensive list of species with a quite high sampling completeness. The active search for specimens in the vegetation proved to be essential in order to have a comprehensive vision of mantid fauna in a given environment. However, the complexity of the vegetation matrix in a tropical rainforest context did not allow us to study the whole volume of available habitats and microhabitats. More sampling would probably result in the discovery of more species restricted to specific ecological niches. Canopy surveying was also very important because many mantids have ecological niches that can be restricted to this stratum. Thus, the verticality of more than $40 \mathrm{~m}$ in an old secondary or in a primary forest is useful to distinguish species with different ecological features. In conclusion, we recommend not restricting future studies to the sole use of light trapping, a by far too much restrictive approach to produce a representative picture of the structure of mantid assemblages.

\section{Acknowledgements}

I wish to thank the Scientific Comittee of "SANGHA Biodiversité en Terre Pygmée" Expedition: Prof. Michel Dron (Institute of Plant Biology, Paris-Saclay), Prof. Jean Laurent Syssa-Magale (Faculty of Science, Bangui), Dr. Thierry Rousselin (Geo212 Company, Paris), Dr. Jean-Marc Seng (Biotransfer Company, Montreuil), Dr. Christian Jost (Research Center on animal cognition), Mr. Jean-Pierre Vidon (Ambassador of France in Bangui), Mr. Daniel Emery Dédé (Deputy, President of Production Committee, Natural Resources and Environment, Chairman of the Parliamentary Group of Friendship-Central Africa Republic-Nigeria), Mr. Karim Mekassoua (Minister of State Sangha-Mbaere, CAR), Mr. Gustave Doungoube and Bob Konzi-Sarambo (Minister of Environment and Ecology of CAR), Dr. Joachim Rouauld, Dr. Serge Florent Bole- 
vane Ouantinam, Dr. Olga Yongo, (University of Bangui), Mr. José Madomi (Minister of Waters and Forests, CAR), Mr. Albert Christian Junior Ndadet (Curator of Dzanga-Sangha Protected Areas, CAR), Mr. Maurice Henri Tadjuide (IUCN at the Tri-National Park), Mr. Jean-Bernard Yarissem (WWF program in CAR), Mr. Sylvain Dongolo, Ms. Angelique Todd (WWF, Bayanga), Mr. François Zowoya (ECOFAC, CAR), Mr. Kazanji Mirdad (Pasteur Institute, Bangui).

Many thanks to Samori Mawendé, spiritual healer Pygmy and all of his community; and all of the team from Central Africa who helped us in the different expeditions.

Sampling in the canopy was made possible thanks to the association "Tout Là-Haut" represented by Matias Loubes and Marion Courcelle; and logistical help in Bangui was provided by Mr. JeanLouis Fijalkowski. This study has been partly funded by the Grant Germaine Cousin $(2012,2014)$ from the Société Entomologique de France, by the Crowdfunding KissKissBankBank of April 2015 "A la recherche de la Biodiversité des Mantes d'Afrique" (Actions Biodiversité NGO, Sergej Buchet and Viviane Calaora, Alain Duhamel, Patrick Moulin and Colette Moulin-Berger), by a parternship with the engineering company "Alise Environnement". DNA barcoding was supported by the International Barcode of Life project led by Paul Hebert at Biodiversity Institute of Ontario (Guelph, Canada). Further, we wish to thank the managers and contributors of the Mantodea Species File (MSF, http://mantodea.speciesfile.org) for the great and useful work they carry out for dictyopterists worldwide, and Roger Roy (Museum National d'Histoire Naturelle, Paris) for his trust and his help in identifying some specimens.

\section{References}

Aveling C, Debonnet G (2010) Le Patrimoine mondial dans le bassin du Congo. Centre du Patrimoine mondial. UNESCO, Paris.

Basset Y (2001) Invertebrates in the canopy of tropical rain forests. How much do we really know? Plant Ecology 153: 87-107. https://doi. org/10.1023/A:1017581406101

Basset Y, Kitching R, Miller S, Novotny V (2003) Arthropods of Tropical Forest: Spatio-temporal Dynamics and Resource Use in the Canopy. Cambridge University Press, Cambridge.

Brugiere D, Sakom D, Gautier-Hion A (2005) The conservation significance of the proposed Mbaéré-Bodingué National Park, Central African Republic, with special emphasis on its primate community. Biodiversity and Conservation 14: 505-522. https://doi.org/10.1007/ s10531-004-3915-6

Chao A, Jost L (2012) Coverage-based rarefaction and extrapolation: standardizing samples by completeness rather than size. Ecology 93: 2533-2547. https://doi.org/10.1890/11-1952.1

Couturier G, Boussienguet J, Dosso H (1986) Recherche entomologique dans les écosystèmes forestiers africains : rapport de l'atelier régional. Atelier Régional IET, ORSTOM, UNESCO-MAB, PNUE, RAB.

de Wasseige C, Flynn J, Louppe D, Hiol Hiol F, Mayraux Ph (2014) The Forests of the Congo Basin - State of the Forest 2013. Weyrich, Belgium.

Dial RJ, Ellwood MD, Turner EC, Foster WA (2006) Arthropod abundance, canopy structure, and microclimate in a Bornean Lowland Tropical Rain Forest. Biotropica 38: 643-652. https://doi.org/10.1111/j.17447429.2006.00181.x

Ehrmann R (2002) Mantodea - Gottesanberinnen der Welt. Natur und Tier-Verlag, Münster, 519 pp.

Giglio-Tos E (1927) Das Tierreich. Orthoptera-Mantidae. Walter de Gruyter and Co., Berlin and Leipzig, 707 pp.

Lowman MD, Rinker HB (2004) Forest Canopies. Second Edition. Elsevier Academic Press, Burlington, USA.
Massa B (2013) Diversity of leaf katydids (Orthoptera: Tettigoniidae: Phaneropterinae) of Dzanga-Ndoki National Park, Central African Republic, with selected records from other African countries. Journal of Orthoptera Research 22: 125-152. https://doi. org/10.1665/034.022.0201

Ndarata Massanguet C (2012) Plan de gestion du Parc National MbaéréBodingué : 2011-2020. ECOFAC IV - Composante Forêt de Ngotto, $168 \mathrm{pp}$.

Ngatoua U (1997) Conservation of Biodiversity in the Central African Republic, Yale FandES Bulletin 102.

Oksanen J, Kindt R, Legendre P, O'Hara B, Simpson GL, Stevens MHN, Wagner H (2008) Vegan: Community Ecology Package. R package version 1.13-1.

Otte D, Spearman L (2005) Mantida Species File. Catalog of the Mantids of the World. Association of the Insects Diversity, $489 \mathrm{pp}$.

Otte D, Spearman L, Stiewe MBD (2017) Mantodea Species File Online. Version 5.0/5.0. http://Mantodea.SpeciesFile.org [September 2017]

Ratnasingham S, Hebert PDN (2007) BOLD: The Barcode of Life Data System (www.barcodinglife.org). Molecular Ecology Notes 7: 355364. https://doi.org/10.1111/j.1471-8286.2007.01678.x

Roy R (2009) Nouvelles données sur le genre Junodia Schulthess, 1899 (Mantodea, Hymenopodidae). Bulletin de la Société Entomologique de France 114: 119-127.

Roy R (2010) Mise au point sur le genre Sphodromantis Stål, 1871 (Mantodea, Mantidae). Bulletin de la Société Entomologique de France 115: 345-366.

Roy R (2013a) Mise au point sur le genre Chrysomantis Giglio-Tos, 1915 (Mantodea, Hymenopodidae). Bulletin de la Société Entomologique de France 118: 463-472.

Roy R (2013b) Révision du genre africain Oxypiloidea Schulthess, 1898 (Dictyoptera, Mantodea, Hymenopodidae). Zoosystema 35: 277-359. https://doi.org/10.5252/z2013n3a1

Roy R (2014) A historical review of nomenclature and high-level classification of praying mantises (Mantodea), including a provisional checklist of the names associated to suprageneric ranks. Zootaxa 3797: 9-28. https://doi.org/10.11646/zootaxa.3797.1.5

Roy R, Stiewe M (2014) Révision du genre Galinthias Stål, 1877 (Mantodea, Galinthiadidae). Bulletin de la Société Entomologique de France 119: 199-215.

Svenson GJ, Hardy NB, Cahillwightman HM, Wieland F (2015) Of flowers and twigs: phylogenetic revision of the plant-mimicking praying mantises (Mantodea: Empusidae and Hymenopodidae) with a new suprageneric classification. Systematic Entomology 40: 789-834. https://doi.org/10.1111/syen.12134

Svenson GJ, Whiting MF (2009) Reconstructing the origins of Praying Mantises (Dictyoptera, Mantodea): the roles of Gondwanan vicariance and morphological convergence. Cladistics 25: 468-514. https://doi.org/10.1111/j.1096-0031.2009.00263.x

Tedrow R, Nathan K, Richard N, Svenson GJ (2014) A new species of Dystacta Saussure, 1871 from Nyungwe National Park, Rwanda (Insecta, Mantodea, Dystacinae). Zookeys 410: 1-21. https://doi. org/10.3897/zookeys.410.7053

Tedrow R, Nathan K, Richard N, Svenson GJ (2015) A survey of the praying mantises of Rwanda, including new records (Insecta, Mantodea). Zootaxa 4027: 67-100. https://doi.org/10.11646/ zootaxa.4027.1.3

UNEP (2008) Africa: Atlas of Our Changing Environment. Division of Early Warning and Assessment (DEWA). United Nations Environment Programme (UNEP). Nairobi, Kenya.

Vande Weghe J-P (2004) Forests of Central Africa: Nature and Man. Protea Book House, $367 \mathrm{pp}$.

Wieland F (2013) The phylogenetic system of Mantodea (Insecta: Dictyoptera). Species, Phylogeny and Evolution 3: 3-222. https://doi. org/10.17875/gup2013-711 